\title{
The Science Case for PILOT III: the Nearby Universe
}

\author{
J. S. Lawrence $\mathrm{A}, \mathrm{B}, \mathrm{N}$, M. C. B. Ashley ${ }^{\mathrm{A}}$, J. Bailey ${ }^{\mathrm{A}}$, D. Barrado y Navascues ${ }^{\mathrm{C}}$,

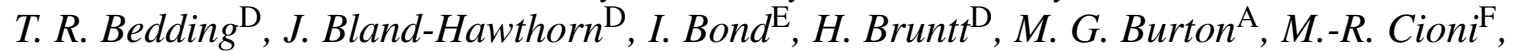 \\ C. Eiroa ${ }^{\mathrm{G}}$, N. Epchtein ${ }^{\mathrm{H}}$, L. Kiss ${ }^{\mathrm{D}}$, P. O. Lagage $^{\mathrm{I}}$, V. Minier ${ }^{\mathrm{I}}$, A. Mora $^{\mathrm{G}}$, K. Olsen $^{\mathrm{J}}$,

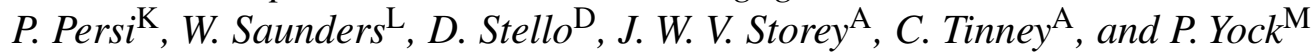 \\ A School of Physics, University of New South Wales, NSW 2052 \\ B Present address: Department of Physics and Engineering, Macquarie University, NSW 2109 \\ and Anglo-Australian Observatory, PO Box 296 Epping, NSW 1710 \\ ${ }^{\mathrm{C}}$ Laboratorio de Astrofísca Espacial y Física Fundamental (INTA), Madrid 28080, Spain \\ D Institute of Astronomy, School of Physics, University of Sydney, NSW 2006 \\ E Massey University, Auckland 0745, New Zealand \\ F Centre for Astrophysics Research, University of Hertfordshire, Hatfield AL10 9AB, UK \\ G Universidad Autónoma de Madrid, C-XI, Madrid 28049, Spain \\ ${ }^{\mathrm{H}}$ CNRS-Fizeau/UNSA, Nice 06108, France \\ I Service d'Astrophysique, CEA Saclay, Saclay 91191, France \\ ${ }^{\mathrm{J}}$ Kitt Peak National Observatory, National Optical Astronomy Observatory, \\ Tucson, AZ 85719, USA \\ K Istituto Astrofisica Spaziale e Fisica Cosmica/INAF, Roma 00100, Italy \\ L Anglo-Australian Observatory, NSW 1710 \\ ${ }^{\mathrm{M}}$ University of Auckland, Auckland 1142, New Zealand \\ ${ }^{\mathrm{N}}$ Corresponding author. Email: jsl@ physics.mq.edu.au
}

Received 2008 November 25, accepted 2009 May 13

\begin{abstract}
PILOT (the Pathfinder for an International Large Optical Telescope) is a proposed 2.5-m optical/ infrared telescope to be located at Dome $\mathrm{C}$ on the Antarctic plateau. The atmospheric conditions at Dome $\mathrm{C}$ deliver a high sensitivity, high photometric precision, wide-field, high spatial resolution, and high-cadence imaging capability to the PILOT telescope. These capabilities enable a unique scientific potential for PILOT, which is addressed in this series of papers. The current paper presents a series of projects dealing with the nearby Universe that have been identified as key science drivers for the PILOT facility. Several projects are proposed that examine stellar populations in nearby galaxies and stellar clusters in order to gain insight into the formation and evolution processes of galaxies and stars. A series of projects will investigate the molecular phase of the Galaxy and explore the ecology of star formation, and investigate the formation processes of stellar and planetary systems. Three projects in the field of exoplanet science are proposed: a search for free-floating low-mass planets and dwarfs, a program of follow-up observations of gravitational microlensing events, and a study of infrared light-curves for previously discovered exoplanets. Three projects are also proposed in the field of planetary and space science: optical and near-infrared studies aimed at characterising planetary atmospheres, a study of coronal mass ejections from the Sun, and a monitoring program searching for small-scale Low Earth Orbit satellite debris items.
\end{abstract}

Keywords: Local Group — galaxies: general — planetary systems: formation — planets and satellites: general — stars: formation — stars: oscillations — telescopes

\section{Introduction}

PILOT (Pathfinder for an International Large Optical Telescope) is proposed as a high spatial resolution wide-field telescope with an optical design and an instrument suite that are matched to the Dome $\mathrm{C}$ atmospheric conditions. These conditions have been shown to offer high infrared sensitivity, due to the low atmospheric thermal emission and low water-vapour column-density (Lawrence 2004; Walden et al. 2005; Tomasi et al. 2006); high spatial resolution and high photometric precision, due to the unique atmospheric turbulence structure above the site (Lawrence et al. 2004; Agabi et al. 2006; Kenyon et al. 2006; Trinquet et al. 2008); and a high cadence, due to the high latitude of the site and the high percentage of cloud-free conditions (Kenyon \& Storey 2006; Mosser \& Aristidi 2007).

The scientific justification for the PILOT telescope has evolved from earlier work (Burton et al. 1994, 2005; Burton, Storey \& Ashley 2001) in parallel with the telescope and instrument suite design (Saunders et al. 2008a,b). The PILOT science case is presented here in a series of three 
papers. Paper I (Lawrence et al. 2009a) gives a summary of the science case, and an overview of the project (including the telescope design, expected performance, and observing strategies). Paper II (Lawrence et al. 2009b) presents a series of science projects for the PILOT facility that are aimed at observing and understanding the distant Universe (i.e. beyond a redshift of $z \approx 1$ ). The current paper discusses PILOT science projects dealing with the nearby Universe (i.e. the Solar System, the Milky Way, and nearby galaxies).

The baseline optical design for PILOT, described in Saunders et al. (2008a), comprises a 2.5-m RitcheyChretien telescope with an $f / 10$ overall focal ratio. The telescope is housed in a temperature- and humiditycontrolled dome that is mounted on top of a $\sim 30$-m high tower in order to elevate the main mirror above the majority of the intense ground-layer turbulence. A fast tip-tilt secondary mirror is used for guiding and to remove residual boundary-layer turbulence and tower wind-shake. As detailed in Paper I (see also Saunders et al. 2008b) the baseline instrument suite for PILOT includes the PILOT VISible Camera (PVISC), a wide-field $\left(40^{\prime} \times 40^{\prime}\right)$ optical imaging camera with a spatial resolution of $\sim 0.3^{\prime \prime}$ over the wavelength range $0.4-1 \mu \mathrm{m}$; the PILOT NearInfraRed Camera (PNIRC), a wide-field $\left(10^{\prime} \times 10^{\prime}\right)$ nearinfrared camera achieving a similar spatial resolution over the wavelength range $1-5 \mu \mathrm{m}$; the PILOT MidInfraRed Imaging Spectrometer (PMIRIS), a wide-field $\left(14^{\prime} \times 14^{\prime}\right)$ mid-infrared instrument operating from 7 to $40 \mu \mathrm{m}$, with several spectral-resolution modes; and the PILOT Lucky Imaging Camera (PLIC), a fast optical camera for diffraction-limited imaging over relatively small-fields $\left(0.5^{\prime} \times 0.5^{\prime}\right)$ in the visible.

The 'nearby Universe' science cases described in this paper are divided into four key themes: stellar properties and populations, star and planet formation, exoplanet science, and solar system and space science. The projects proposed under these themes have been identified to take advantage of the unique discovery space of the PILOT telescope that is enabled by the Dome $\mathrm{C}$ site conditions and the telescope design. For each project, the context and impact is discussed, along with the capabilities of competing (and synergistic) facilities. Additionally, the observational requirements are identified. It is shown that the observational requirements for these projects can either only be achieved with PILOT or can be achieved with other facilities but with greatly reduced efficiency. While many of the science projects described here, and in Paper II, require large amounts of observing time (several seasons in some cases), the observing strategies proposed in Paper I should allow the majority of these science projects to be accomplished within the proposed ten-year lifetime of the PILOT facility.

The stellar properties and populations theme (Section 2) links several projects that examine nearby galaxies and stellar clusters. The first project involves a study of disc galaxies, in the optical and infrared, out to the edge of the Local Group. This study aims to examine the relationship between stellar populations, star formation history, and the local environment. A second project aims to examine the stellar content of the outer regions of (nearer) satellite galaxies over wider fields, to gain insight into the processes of galaxy formation and evolution. A project to image the Magellanic Clouds to a greater depth with higher spatial resolution than existing surveys aims to understand star formation processes and extreme populations of AGB stars. Finally, a project involving long time-series optical observations of nearby globular and open clusters aims to study age-metallicity relationships, test various predictions of stellar astrophysics, and improve our understanding of the physics of massive stars.

The star and planet formation theme (Section 3) links several projects that deal with the early evolutionary stages of stellar and planetary systems. A wide-area survey of the Milky Way Central Molecular Zone in the mid-infrared lines of molecular hydrogen is proposed in order to understand the life history of the molecular phase of the Galaxy. A survey of the Chamaeleon dark clouds complex at several lines in the mid-infrared aims to explore planet formation via the identification of circumstellar discs around young low-mass stellar objects and young brown dwarfs. We also discuss here the potential to investigate the onset of planet formation in protoplanetary discs via the midinfrared spectral signatures of cyrstalline silicates, and the potential to investigate the very early stages of star formation via the mid-infrared photometric characterisation of embedded young stellar objects.

Three exoplanet science studies are proposed in Section 4. The first project involves a near-infrared search for planets 'free-floating' in nearby star clusters, with the aim of directly detecting objects down to a few Jupiter masses. The second project involves a program for optical follow-up of gravitational microlensing candidates based on alerts from dedicated survey telescope networks; this is aimed in particular at firmly determining the abundance of ice-giant planets. Finally, a project to obtain highprecision near- and mid-infrared photometric light-curves of previously discovered exoplanets aims to characterise the atmospheric properties of a large number of hot Jupiters.

The solar system and space science theme (Section 5) deals with several Solar System projects. The first project will investigate atmospheric and surface properties of Mars and Venus via high spatial resolution imaging in the visible and near-infrared. The second project proposes to observe the Sun at mid-infrared and sub-millimetre wavelengths to investigate the physical mechanisms responsible for solar coronal mass ejections. The final project aims to identify accurate orbits for most of the small-scale Low Earth Orbiting space debris.

\section{Stellar Properties and Populations}

\subsection{Stellar Populations in Local Group Galaxies}

Here, we present a science case for deep imaging of stellar populations with PILOT over degree-sized fields-of-view 
with an effective 'seeing' substantially better than the best sites today.

These capabilities are essential to tracing stars in crowded regions, and in the outer parts of galaxies to separate stars from background galaxies. In recent years, stellar populations in nearby galaxies have begun to reveal tantalizing clues to their formation. Continued success in this field demands that we reach magnitude limits comparable to the Hubble Space Telescope but over much larger fields-of-view. It is essential to observe at a range of distances, from $\sim 1$ out to at least $10 \mathrm{Mpc}$, in order to cover a sufficient range in local density contrast with respect to the cold dark matter background, with still greater gains if we can reach the Virgo cluster. Only then can we relate the richness of stellar populations and star formation history to the environment.

An optical and near-infrared survey of Local Group galaxies with PILOT at a level of detail far beyond the reach of current theoretical simulations will provide an observational view of the basic galaxy formation physics that can complement and provide input into simulations of galaxy formation at high redshift. Such a survey will also provide fundamental insight on the process of local galaxy formation. Will we find the edge or will the stellar disc extend even beyond the HI disc? Just how far do baryons extend within the dark matter halo? How extended are bulges in galaxies beyond the Local Group, and do bulge properties have influence on the discs? There are no reliable predictions to date.

\subsubsection{Discs, Bulges and Halos in Nearby Galaxies}

An ongoing fundamental question in astrophysics is: how do disc galaxies form and how do they evolve through cosmic time? There have been important new observations concerning discs and spheroids in recent years showing that there is still a great deal to learn. Recent baryon inventories, for example, suggest that most stars are in spheroids today (e.g. Fukugita, Hogan \& Peebles 1998), while proper bulge/disc deconvolution reveals that most stars are now in discs and, furthermore, that discs account for a large fraction of the red light in galaxies (Benson et al. 2007; Driver et al. 2007). Discs are also the most fragile of galaxy components, and hence the most sensitive to the physics of galaxy formation (Robertson et al. 2006). Therefore, discs lie at the forefront of galaxy formation and evolution studies.

Resolved stellar populations have now been studied in four galaxies (the Milky Way, M31, M33, and NGC 300). Three of these discs (the Milky Way, M31, NGC 300) show no evidence of truncation out to 10 optical scale lengths or more (Worthey et al. 2005; Yong et al. 2006; Vlajic et al. 2008). Pohlen et al. (2008) have shown that outer discs appear to come in three flavours: endlessly exponential, truncated or flattening off. These observations were established with surface photometry and remain to be verified with star count observations of a large survey sample.

Bulges are intimately linked to discs. Bulges are thought to primarily form either from early monolithic collapse (Eggen, Lynden-Bell \& Sandage 1962), through major mergers (Kauffman et al. 1994), or through disc instabilities during the rapid early build-up of the discs (Bower et al. 2006). Bulges provide the link between disc and elliptical galaxies, as they share similar surface brightness distributions and predominantly red colours with ellipticals, indicating their dominance by old stellar populations. Thus, understanding the properties of bulges in comparison and contrast to discs and elliptical galaxies is a crucial step towards a unified picture of galaxy formation.

Substructure in galaxy haloes is a topic of enormous current interest. A remarkable result was the discovery of the Sgr stream (Ibata, Gilmore \& Irwin 1994), which is now known to account for about half the stellar mass of the Galactic halo. Deep observations of M31 and other nearby discs have revealed spectacular streams in the outer haloes (e.g. Ferguson et al. 2002), thought to represent the satellite accretion histories of massive galaxies (e.g. Bullock \& Johnston 2005; Helmi 2008). Whether satellite accretion is responsible for nearly all halo formation, and the degree to which satellite accretion contributes to disc assembly, remains unknown.

\subsubsection{Sensitivity and Crowding}

Stellar photometry depends critically on an accurate measure of the background, fluctuations in which impose a fundamental limit on the possible accuracy of the photometry. In Figure 1, the crowding limits for various target galaxies observed with the PLIC, PVISC, and PNIRC cameras of PILOT are estimated following the analysis of Olsen et al. (2003). The crowding limit is defined to be the magnitude at which the photometric error due to crowding is 0.1 magnitudes. This limit depends on the telescope resolution, the magnitude of the target star, and the distance, surface brightness, and luminosity function of the star's environment. For the purposes of this investigation, we assumed that all target galaxies have surface brightness profiles in their outer disks like that found by Irwin et al. (2005) and Ibata et al. (2007) along the minor axis of M31, who measured surface brightnesses between 25 and 29 mag $\operatorname{arcsec}^{-2}$ at $V$ band at radii between 8 and $18 \mathrm{kpc}$ from the center.

With the high resolution Lucky Imaging camera, PLIC, PILOT will achieve photometry in nearby galaxies as deep as with HST/ACS. In the outskirts of the Magellanic Clouds, PLIC will reach the bottom of the stellar luminosity function, whereas in Sculptor group galaxies, such as NGC 300, the photometry will reach well below the level of the horizontal branch. The confusion limits for PVISC are similar to those of PLIC at much lower surface brightnesses, reaching the level of the horizontal branch out to $\sim 3.5 \mathrm{Mpc}$. At distances of $\sim 7 \mathrm{Mpc}$, PVISC will still resolve stars down to below the red giant branch tip. PILOT+PVISC, with its large field and excellent resolution, will thus be a powerful instrument for mapping the properties of extended galaxy discs and halos. Observing in the near-infrared with PNIRC makes it possible to resolve red giants out to $\sim 10 \mathrm{Mpc}$, and AGB stars out to 

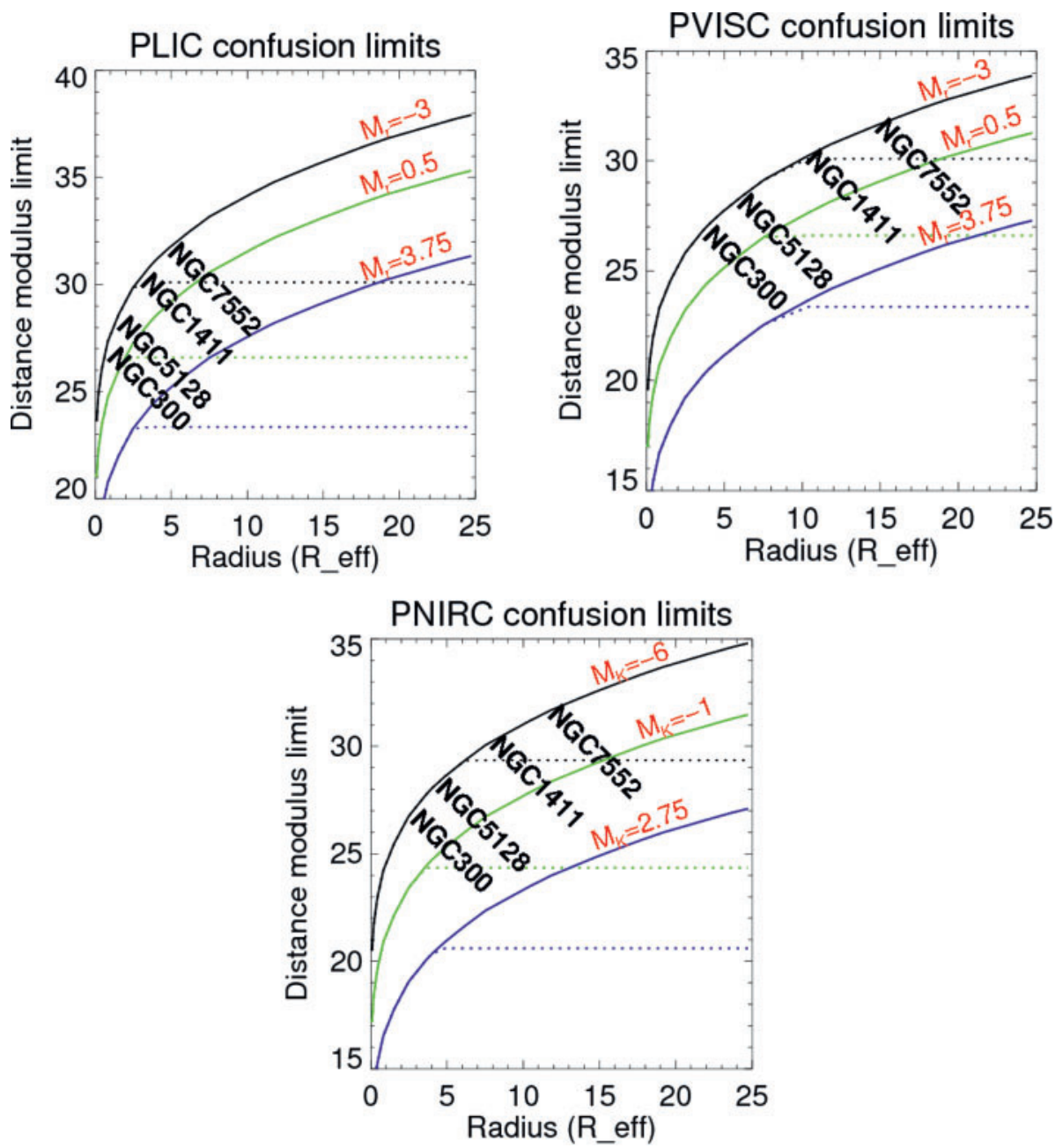

Figure 1 Confusion depths for PILOT with PLIC, PNIRC, and PVISC cameras. In these figures, we explore the ability of PILOT to resolve stars with selected intrinsic luminosities, assuming that the target galaxies have surface brightness profiles like that found by Irwin et al. (2005) and Ibata et al. (2007) along the minor axis of M31 (with $R_{\mathrm{eff}}=0.1^{\circ}$ ). The solid curves show the distances at which photometric error due to confusion would reach $10 \%$, as a function of radius along the assumed surface brightness profile. Individual curves are shown for RGB tip stars (black lines), horizontal branch stars (green lines), and main sequence turnoff stars (blue lines), assuming an age of $10 \mathrm{Gyr}$ and metal abundance $Z=0.0001$ and the Girardi et al. (2002) isochrones. The confusion limits were calculated following Olsen et al. (2003), by relating the photometric error induced by confusion to the telescope resolution, the magnitude of the target star, and the distance, surface brightness, and luminosity function of the star's environment. Dotted lines indicate the distances at which photon noise error in a 1-hour exposure would exceed the error due to crowding.

the Virgo cluster, owing to the very high luminosities of these stars in the near-infrared.

\subsubsection{Observational Approach}

A program of deep, wide-field imaging of a sample of galaxies out to $\sim 10 \mathrm{Mpc}$ would allow us to answer a number of very exciting questions. We would aim to understand the relationship between extended discs, inner discs, bulges, and halos, and to control for the cosmologically predicted effect of the density of the environment.

The program is challenging for many reasons, however. First, we need to reach a photometric depth sufficient to determine metallicities from stars a few magnitudes down the metal-poor red giant branch; to push beyond the Local Group, we must reach a $(5-10 \sigma)$ point source sensitivity to $m_{\mathrm{AB}} \approx 28$ in $g$ and $r$ bands, and $m_{\mathrm{AB}} \approx 27$ in the nearinfrared at $K$ band. It must be stressed that accurate stellar photometry below this limit in any band is very challenging. Excellent seeing, stable photometric conditions, and a dark site are essential, as are high instrument throughput and a stable flatfield. Second, the need for global coverage and the extents of the target galaxies requires wide fields in order to map several square degrees per galaxy. Finally, for galaxies at these distances, crowding by point sources is a serious limiting factor under conventional seeing conditions, even in the low surface brightness extended discs.

The goal of this study is to observe a sample of disc galaxies over a range of environmental density. We will use PLIC to study the high surface brightness inner parts of the discs, and PVISC and PNIRC to map the low 
surface brightness extended discs. PVISC observations reaching down to the horizontal branch in galaxies within $\sim 3.5 \mathrm{Mpc}$ (and thus chiefly low density environments) will allow us to study the detailed star formation histories of extended discs, while we will use PVISC and PNIRC observations of red giant and AGB stars in more distant galaxies to map the prevalence and extent of such discs in many different environments. We will primarily target moderately inclined and face-on discs at reasonably high Galactic latitude, as these avoid projection effects in our analysis of population gradients and minimize Milky Way foreground extinction. We also need to observe as many galaxies as possible within each environment, to control for the variance in the interaction histories of the galaxies.

The actual magnitude limit is a shallow function of distance, as the idea is to reach the limit imposed by confusion in each galaxy. For an accurate metallicity determination and star/galaxy discrimination, 3 or 4 filters, likely to be $g, r, J, K_{\mathrm{d}}$, will be required for each galaxy; the exact choice will depend on the particular galaxy to be studied. To detect the TRGB out to $10 \mathrm{Mpc}$ in $g$ and $r$ will require $\sim 1$ hour with PVISC, and in $J$ and $K$ will require $\sim 3.5$ hours with PNIRC. Detecting the horizontal branch out to $4 \mathrm{Mpc}$ will require $\sim 10$ hours of exposure with PVISC in $g$ and $r$, and $\sim 1$ hour with PNRIC in $J$ and $K$ to get $\sim 2$ magnitudes below the TRGB. Our goal is to sample the populations in the disks out to $10-15 R_{\text {eff }}$, the radius out to which M31's disk has been traced (Irwin et al. 2005; Ibata et al. 2007). For galaxies with distances $1.5<d<10 \mathrm{Mpc}$, we can capture the necessary area with one PVISC pointing, whereas with PNIRC we would use $\sim 3$ pointings per galaxy to probe the range of radii. Guided by the Tully et al. (2005) catalog of nearby galaxies, we find that PILOT will have 22 possible targets for TRGB measurement and 10 targets for horizontal branch measurement. Given the rough exposure times and number of pointings above, we could observe the entire sample of 32 galaxies in $g, r, J, K_{\mathrm{d}}$, in $\sim 800$ hours during one complete winter season.

The science case outlined here demands a telescope with excellent resolution for overcoming stellar crowding, and a wide field for mapping and characterizing extended stellar discs in galaxies over a range of environments. The Antarctic site conditions, and the PILOT telescope in particular, provide a very good match to these requirements. These studies are important for understanding how disc galaxies formed, as they track the build-up of stellar mass, and place limits on the merger histories of disc galaxies. They do not, however, have the resolution to study high surface brightness discs beyond distances of $\sim 4 \mathrm{Mpc}$, and thus do not reach the richest galaxy environments. The recent discovery of extremely low surface brightness extended stellar discs is thus very exciting. Not only do they offer a fresh perspective on the formation history of disc galaxies generally, but they give us the opportunity to trace stellar disc formation out to larger distances and thus richer environments, where we expect to be able to see differences in the hierarchical build-up of galaxies compared to those in the Local Group.

\subsection{Outer Structures of Satellite Galaxies}

This project aims to observe the stellar content of the outer regions of nearby Milky Way satellite galaxies (out to $\sim 600 \mathrm{kpc}$ ) that are sufficiently nearby to allow for their individual members to be resolved. Galaxies with a resolvable stellar content provide a useful tool to understand the process of galaxy formation and evolution. Tracing the distribution of stars of a different type, age, and metallicity allows us to access the propagation of the star formation history throughout galaxies and the morphology of galaxies as a function of time.

The outermost parts of galaxies harbour faint and old stars that, according to cosmological simulations, may be associated with a galaxy halo and bear the signature of tidal streams resulting from galaxy interaction and satellite accretion events. Even the halos of those galaxies that did not experience major mergers preserve the fossils of their formation history which can be revealed by widefield sensitive studies of halo objects such as red giant and horizontal branch stars. On the contrary, galaxy halos may be rich in substructures like stellar clusters. One of the challenges of modern astronomy is to understand if stellar clusters with multiple stellar populations are the relics of dwarf galaxies that were accreted at earlier times.

This project aims to observe the outer regions of galaxies that are sufficiently nearby to allow for individual field and cluster members to be resolved. The main goal is tracing the outer morphology, structure, age, and metallicity of galaxies. This will have a direct impact on our understanding of the process of galaxy formation and evolution. It will complement current studies on the most crowded, active and central regions of galaxies (e.g. Holtzman, Smith \& Grillmair 2000) addressing, for example, issues of star formation. It also provides a complement to the Local Group galaxy survey proposed in the previous section, as it will provide greater detail on the outer structures of closer galaxies.

The outer regions surrounding nearby galaxies have been mostly studied in a limited number of fields (e.g. Demers, Battinelli \& Artigau 2006; Gullieuszik et al. 2007). These investigations successfully probed the presence of an old stellar population which is much more extended than initially expected (Majewski et al. 2005; Munoz et al. 2006), as illustrated in Figure 2. At present, and for many more years to come, VISTA will be the only type of facility that can be used for these studies. Its spatial resolution $\left(\sim 0.34^{\prime \prime}\right.$ per pixel for a natural seeing of at best $\left.\sim 0.6^{\prime \prime}\right)$ is the only limitation restricting the galaxies that this study can probe out to a given distance. The small field-of-view of planned infrared instruments for ELTs and JWST precludes their use for wide-area survey projects.

The major contribution of PILOT to this research will be the combination of high spatial resolution and wide-field coverage at near-infrared wavelengths. The 


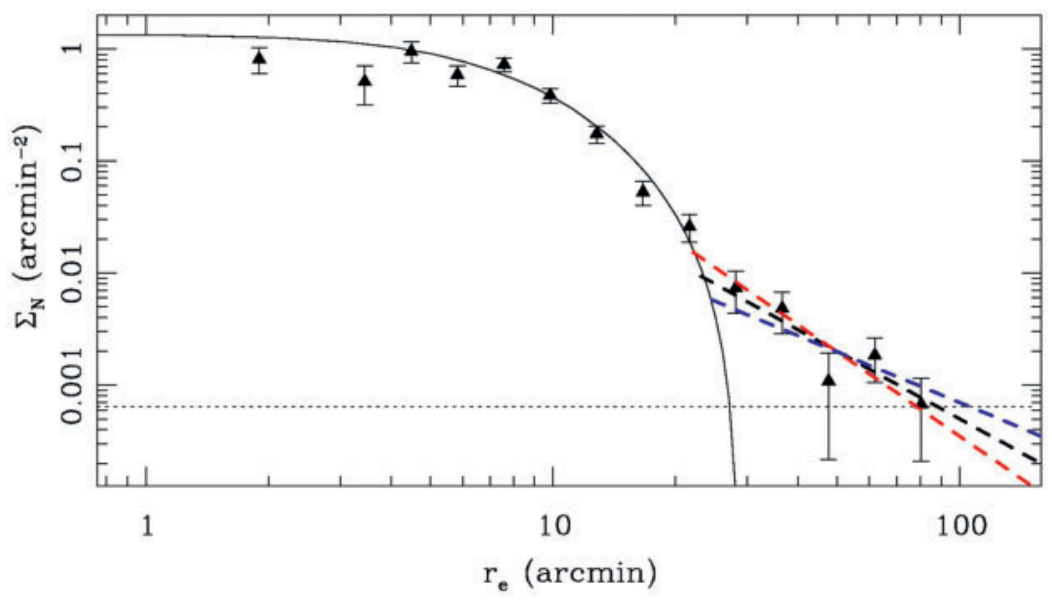

Figure 2 Density profile for the Carina dSph galaxy showing extended stellar components at large radii. From Munoz et al. (2006). PILOT will enable the stellar content in these outer regions of such galaxies to be analysed in more detail than currently possible.

possibility of exploiting a natural seeing that is considerably better than that available for a similar wide-field instrument at other ground-based site will allow us to target galaxies that are further away and, most importantly, to distinguish fainter sources in their outskirts.

The old and extended stellar population of galaxies is traced either by red giant branch (RGB) stars or by RR Lyrae variable stars. RGB stars are usually more numerous than RR Lyrae stars and from the bottom of the RGB to the tip of the RGB they span $\sim 8$ mag in the $K$ band. RR Lyrae stars have a luminosity which is about 6 magnitudes fainter than the tip of the RGB.

RR Lyrae stars are easily identified in optical wavebands from their light-curve. In the $K$ band their amplitude of variation is reduced and with just a limited number of epochs it is possible to obtain a very good estimate of their mean magnitude. The period-luminosity relation for RR Lyrae stars in the $K$ band has a small scatter and depends very little on age and metallicity providing a very powerful distance indicator (Pietrzynski et al. 2008). The combined number of epochs that will be used for the study of RR Lyrae stars will also be sufficient to detect most RGB stars.

The 1 hour sensitivity limit with PILOT is $m_{\mathrm{AB}}=25.3$ at $K_{\mathrm{d}}$ for $5 \sigma$ (which is the minimum SNR required for this study). This would allow the detection of the entire RGB and RR Lyrae stars in galaxies out to distances of $\sim 600 \mathrm{kpc}$ where we find most of the satellites of the Milky Way. The Magellanic Clouds are being extensively studied with other facilities such as VISTA in the $Y, J$, and $K_{\mathrm{S}}$ wavebands via the VMC survey; a deeper and higher wavelength study of the Magellanic Clouds is proposed for PILOT in the next Section. The remaining Milky Way satellites span a range of morphological types and histories that are perfectly suitable for this study. Targets within $600 \mathrm{kpc}$ include Sculptor, Carina, Fornax, Phoenix, and NGC 6822. The main body of these galaxies covers an angular scale ranging from $5^{\prime} \times 4^{\prime}$ to $40^{\prime} \times 31^{\prime}$.

To survey each of these galaxies out to three times the extent of the galaxy in both directions will require a total coverage of $\sim 5 \mathrm{deg}^{2}$. This equates to $\sim 180$ fields with
PNIRC. Multi-band observations in at least $Y, J$, and $K_{\mathrm{d}}$ filters, and multiple observations of each field at different times are required. The indicative total observing time for this project is $\sim 4000$ hours (i.e. $\sim 2$ winter seasons).

\subsection{A Magellanic Clouds Broadband Survey}

The Magellanic Clouds (MCs) offer a unique opportunity to investigate in great detail - at the level of individual stars - the history of the evolution of a complex system of gas and stars and the recycling of matter within a relatively limited area of the sky (about $100 \mathrm{deg}^{2}$ ) where the distance is accurately known.

The programme proposed here for PILOT is to map fully and uniformly the surface of both MCs and selected areas of the Magellanic Stream at an unprecedented level of sensitivity and angular resolution in the $K_{\mathrm{d}}$ and $L_{\mathrm{s}}$, and possibly the $L^{\prime}$ and $M^{\prime}$, bands. This will complement and considerably enhance the database already obtained with 2MASS/DENIS and the InfraRed Survey Facility (IRSF) in the non-thermal infrared (up to $K_{\mathrm{S}}$ ), and Spitzer (SAGE) in the mid- and far-infrared, or planned to be obtained with VISTA (VMC) up to $K_{\mathrm{s}}$. This survey will basically investigate:

1. The star-formation processes in an environment of lower metal abundance than in our Galaxy and subject to strong tidal effect from our Galaxy, and

2. Extreme populations of AGB stars characterized by a high mass loss rate and thick circumstellar envelopes that may have escaped detection by earlier deep 2- $\mu \mathrm{m}$ surveys or not resolved enough or confusion limited in the SAGE survey.

This project relies on a number of the advantages that the Dome $\mathrm{C}$ location offers with respect to conventional sites. It requires the exceptional observing conditions in the near thermal infrared (improved transmission, low sky emissivity by a factor of up to 3 magnitudes at $K_{\mathrm{d}}$, better photometric stability, and the opportunity to open new windows such as the $L_{\mathrm{s}}$ band at $3-3.5 \mu \mathrm{m}$ ) and the excellent seeing conditions $\left(0.3^{\prime \prime}\right)$ above the 
turbulent boundary layer. Additionally, observing efficiency is increased thanks to the very low background emission of the sky throughout the year; observations may even be possible in summer time, at least in the $L_{\mathrm{s}}$ filter and possibly fractions of the summer 'days' in the $K_{\mathrm{d}}$ window. Finally, the high latitude of Dome $\mathrm{C}$ means that the MCs are circumpolar and thus can be observed all year round at small and almost constant airmass (1.0 to 1.15).

This Magellanic Clouds study is complementary to the 2MASS/DENIS, IRSF and future VISTA Surveys. In the last decade, several near-infrared surveys of the Magellanic Clouds have been performed providing catalogues of millions of stellar entries, in the $I, J, H$, and $K$ bands. These have included DENIS (Cioni et al. 2000), 2MASS (Nikolaiev et al. 2000), and IRSF (Kato et al. 2007). Colour-colour diagrams (see for example Figure 3) have been extensively used to separate different populations of stars and objects and, thanks to the fact that the objects are essentially at the same accurately known distance, colourmagnitude diagrams have been used as well, to provide excellent luminosity calibrations of these populations.

The SPIREX telescope at the South Pole indicated the kind of study that would be possible, through deep thermal infrared imaging of the 30 Doradus region of the LMC (Maercker \& Burton 2005).

In addition, the Spitzer Legacy program, Surveying the Agents of a Galaxy's Evolution (SAGE) ${ }^{1}$, has recently provided unique images and catalogues of sources of the MCs (e.g. Whitney et al. 2008) in the mid- and far-infrared. SAGE has undertaken a comprehensive picture of the current star formation activity, which is traced by the IRAC $(3.5,4.5,5.8$, and $8.0 \mu \mathrm{m})$ and $\operatorname{MIPS}(24,70$, and $160 \mu \mathrm{m})$ bands, which have spatial resolutions of $2^{\prime \prime}$ and 6-40" respectively.

Starting in 2008, one of the VISTA Public Surveys, $\mathrm{VMC}^{2}$, will be aimed at deep imaging of the MCs and Bridge. This will provide unprecedented catalogues of faint objects in the $Y, J$, and $K$ bands to $m_{\mathrm{AB}}=22.9,22.8$, and 22.3 respectively at $10 \sigma$ across some $200 \mathrm{deg}^{2}$, at a seeing-limited FWHM resolution of $\sim 0.8^{\prime \prime}$. The total number of nights required to achieve VMC in 5 years is approximately 200. It will resolve stellar populations into old populations, active star formation areas, and ongoing merging. It will globally resolve the star history with unprecedented accuracy and trace past interactions.

The aim of this survey is to cover in a first campaign the $7 \times 7^{\circ}$ area of the LMC at $K_{\mathrm{d}}$ and $L_{\mathrm{s}}$ (with a possible extension to $L^{\prime}$ and $M^{\prime}$ ) at the sensitivity limit expected in one hour with the PILOT Near InfraRed Camera (PNIRC), i.e. $m_{\mathrm{AB}}=25.3$ and 21.2 , respectively, at a spatial resolution of $0.3-0.4^{\prime \prime}$. This is $\sim 15$-times deeper than VISTA at $2.2 \mu \mathrm{m}$, with 2.5 -times the angular resolution, and half as deep as SAGE $(5.1 \mu \mathrm{Jy})$ at $3.6 \mu \mathrm{m}$, but at an angular resolution 5-6 times higher.

\footnotetext{
${ }^{1}$ See http: //sage.stsci.edu/project.php.

2 http: //www.eso.org/sci/observing/policies/ PublicSurveys/sciencePublicSurveys.html.
}
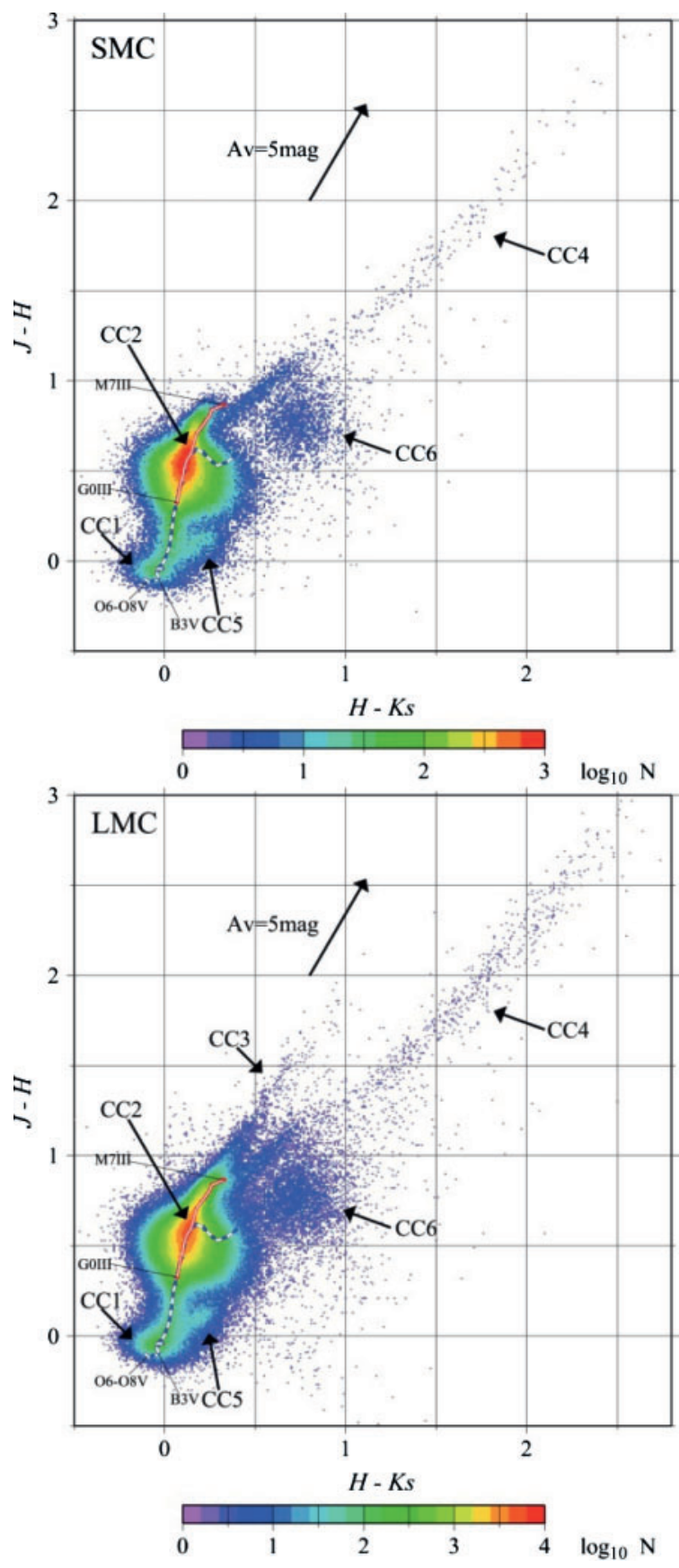

Figure 3 Infrared colour-colour diagram of the Large Magellanic Clouds (top) and the Small Magellanic Clouds (bottom) from the IRSF survey. From Kato et al. (2007). The MC survey proposed here for PILOT would extend to longer wavelengths and deeper limits, allowing star formation to be probed to lower masses in more obscured environments.

This extension will be extremely useful to single out more deeply embedded objects (younger or experiencing even more intense mass loss) that only show up in the $3-5 \mu \mathrm{m}$ range, as well as to pick up objects of lower surface temperatures (e.g. extreme carbon stars). The PILOT MC survey will offer an opportunity to link the VISTA and Spitzer SAGE data, and will prepare for more detailed observations with JWST and/or a southern ELT.

The baseline near-infrared camera for PILOT, PNIRC, is equipped with a single channel near-infrared $4 \mathrm{k} \times 4 \mathrm{k}$ 
array covering a $10^{\prime} \times 10^{\prime}$ field at $0.15^{\prime \prime}$ per pixel. This instrument would require about 2000 hours of observation to cover the 50- $\mathrm{deg}^{2}$ area of the LMC at the ultimate depth ( $m_{\mathrm{AB}} \approx 25$ at $K$ band). This could be achieved in approximately 3 months of continuous and essentially uninterrupted observations taking into account calibration and overheads. Another 1000 hours would be necessary to cover the SMC and some selected fields in the Magellanic Stream at the same level of sensitivity.

The efficiency of this survey is a strong function of the instrument specification. The efficiency would be greatly increased by enlarging the focal plane coverage, either by using larger arrays (i.e. with a mosaic of $4 \mathrm{k} \times 4 \mathrm{k}$ arrays), or a multi-channel configuration (e.g. with $K_{\mathrm{d}}, L_{\mathrm{s}}$, and $L^{\prime}$ arms). The latter option has the advantage of providing simultaneous photometric data, with no phase lag for variable objects, and thus accurate colour indices. The ideal configuration involves a trade off between cost, observing lapse of time, and requested sensitivity. The most important issue is probably to reach the best gain in sensitivity compared to the VISTA survey in the $K$ band.

\subsection{Asteroseismology of Stars in Clusters}

The study of stellar oscillations, known as asteroseismol$o g y$, allows us to probe the interiors of stars. Oscillation periods range from a few minutes up to days or even years, and the photometric amplitudes range from a few parts per million up to a magnitude or more. Asteroseismology is a rapidly growing field that covers a wide range of stars, including Sun-like stars, red giants, hot massive stars and white dwarfs.

Recent results have demonstrated the potential for time-series photometry from Dome C (Rauer, Fruth \& Erikson 2008; Strassmeier et al. 2008). With PILOT, we will be able to apply the techniques of asteroseismology to stars in clusters. The projects described below involve observing oscillating stars in two types of clusters using the PILOT Visible Camera (PVISC), taking advantage of the following capabilities:

- A large field-of-view with strongly reduced crowding problems thanks to the exceptional seeing. Only HST can to do better in resolution, but it has a much smaller field;

- Nearly-continuous temporal coverage, which means improved precision in frequency determination, without the ambiguities associated with one-cycle-per-day aliases;

- A much smaller range of airmasses than at low-latitude sites, since a given field stays at roughly constant elevation as the Earth rotates. This should yield photometry whose precision on time-scales of hours to days is exquisite; and

- Photometric precision will be further improved by the low scintillation noise at Dome $\mathrm{C}$, which should allow us to detect much smaller fluctuations than at any other ground-based observatory.
The importance of asteroseismology is seen in the number of projects underway or being planned. The French/ESA CoRoT space mission is currently collecting data and the NASA Kepler mission was recently launched in March 2009. CoRoT is observing a handful of predetermined fields in its continuous viewing zones ${ }^{3}$, while Kepler will study a single field in Cygnus (with the primary aim of detecting transiting exoplanets ${ }^{4}$ ). Neither has the capability to observe clusters in the way proposed here. On the ground, the desire to obtain continuous coverage has led to plans for global networks of telescopes at temperate sites, to carry out observations in photometry (LCOGT; Hidas et al. 2008) and radial velocity (SONG; Grundahl et al. 2006). Again, these are geared towards individual stars and do not have the capacity to observe clusters. The same applies to the SIAMOIS instrument, which has been proposed for Dome C by Mosser et al. (2007). If funded, it will measure precise stellar velocities and allow asteroseismology of a small number of bright solar-type stars, complementary to, but very distinct from, the observations of huge numbers of much fainter cluster stars, to which PILOT is uniquely suited.

\subsection{1 $\omega$ Centauri Probing Star Formation in the Early Universe}

Globular clusters (GCs) contain $10^{5}-10^{6}$ very old stars within roughly spherical aggregates that are several tens of parsecs in diameter (see Figure 4). Typical ages range from 10 to $13 \mathrm{Gyr}$, with strong chemical and age homogeneity within each cluster. These objects are enigmatic ingredients in stellar and Galaxy formation, offering an opportunity to unravel cosmic history through careful observations. In particular, one current understanding of the formation of the Milky Way Galaxy envisages hierarchical merging of low-mass building blocks that were formed in mini-halos at redshifts $z \approx 5-10$ (e.g. Kravtsov \& Gnedin 2005; Bekki et al. 2007). Within this framework the 150 or so globular clusters in the Milky Way system (Harris 1996) are thought to represent the survivors from these original building blocks. The small range of metallicities in individual clusters and the large clusterto-cluster variations could indicate a chemical diversity among the mini-halos reflecting the cosmic variance of initial conditions of star formation.

The discovery of multiple stellar populations in $\omega$ Cen, the most massive and exceptional GC of the Milky Way (Lee et al. 1999), together with the fact that the second most massive GC (M54) is a core of the disrupting Sagittarius dwarf galaxy (Layden \& Sarajedini 2000), have strengthened the view that some of the most massive GCs might be the remnant cores of disrupted nucleated dwarf galaxies. The peculiar colour-magnitude diagram of $\omega$ Cen (see Figure 5) contains multiple branches, indicating a mixture of distinct stellar populations within the cluster. Two basic scenarios have been proposed to

\footnotetext{
${ }^{3}$ See http: //smsc.cnes.fr/COROT/.

${ }^{4}$ See http: //kepler.nasa.gov/.
} 

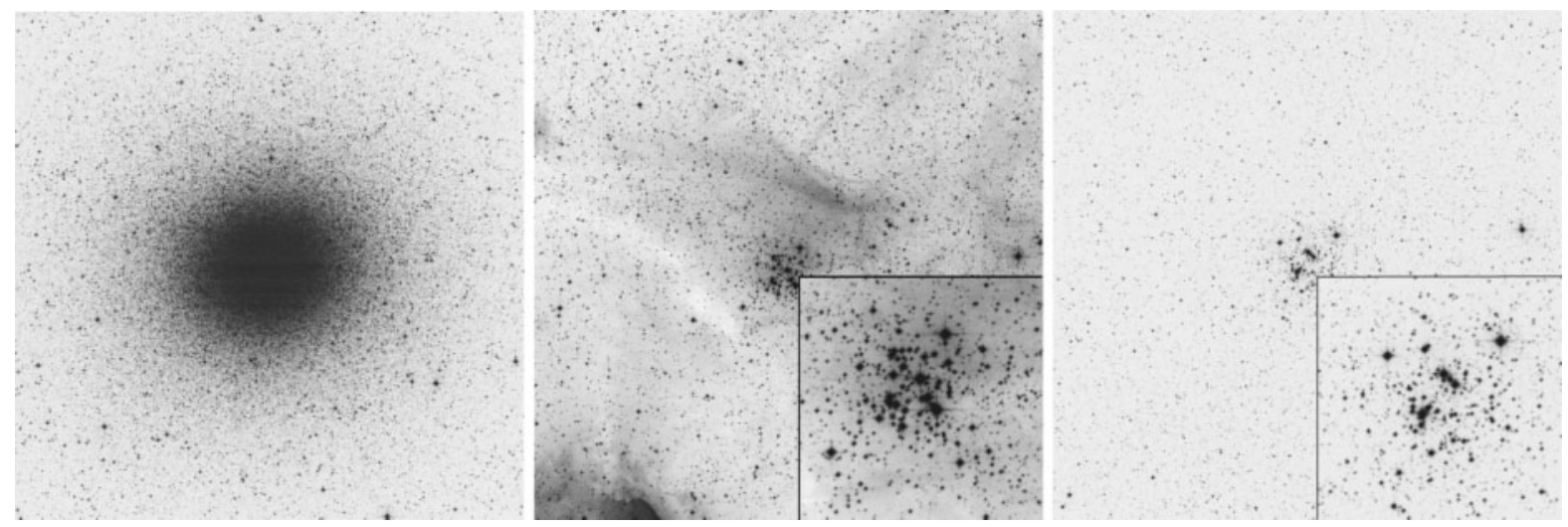

Figure 4 The field-of-view of PILOT is perfect for $\omega$ Cen (left panel) and will fully cover both NGC 3293 (middle) and NGC 4755 (right). For NGC 3293 and NGC 4755 an expanded view $(\times 2)$ of the central part of the cluster is shown in the lower right part of the panel. The three images are all $42^{\prime} \times 42^{\prime}$ ( the FOV of PVISC) and obtained from the Digitized Sky Survey. Note that the field-of-view of WFPC3, to be installed on HST in 2009 , is $2.7^{\prime} \times 2.7^{\prime}(0.4 \%$ of the area covered by PILOT).

explain the spread in chemical composition and the other unusual properties of $\omega$ Cen. The first is self-enrichment from the first-generation asymptotic giant branch stars and Type II supernova events, either as an isolated cluster or as the nucleus of a dwarf galaxy. The second scenario proposes some sort of merger event, such as a merger between two or more globular clusters or between a dwarf galaxy and a globular cluster. There has been a great interest in testing these scenarios and the evidence is rather inconclusive (e.g. Platais et al. 2003; Pancino et al. 2007).

We propose a new approach to investigate the properties of distinct populations, using asteroseismology in combination with photometry and spectroscopy to derive accurate stellar parameters along the red giant and subgiant branches of $\omega$ Cen. In particular, asteroseismology will give us constraints on the stellar age to about $10 \%$ and the mean stellar density to less than $1 \%$ (Kjeldsen et al. 2009). We will use the unique capabilities of PILOT to obtain a long time-series of sharp images. The proposed $0.5-\mathrm{deg}^{2}$ field-of-view of the PILOT PVISC camera will allow us to capture millions of stars in $\omega$ Cen, of which several tens of thousands will oscillate with measurable amplitudes and periods. Our main targets will be the bright red giants, for which the predicted periods range from several hours to days. To achieve our goals, we will need about one month of reasonably continuous observations (targetof-opportunity breaks are acceptable). Observations will be principally in the $r$ band, with exposures probing the range 13-16 mag. The high photometric precision will allow us to probe less luminous stars, further down on the multiple red giant branches.

Accurate stellar parameters from asteroseismology should enable us to resolve the degeneracy between age, mass and helium abundance, and to test directly whether more metal-rich stars are younger, as predicted by the scenarios of prolonged star formation. Comparing individual frequencies of the various groups of stars in $\omega$ Cen with stellar pulsation models will also enable us to investigate the metallicity dependence of the oscillations.
Stars in $\omega$ Cen can also be used to test predictions of stellar astrophysics, a significant result alone added to the studies of the cluster as a system. As an added bonus, $\omega$ Cen contains thousands of classical pulsating stars, including large amplitude RR Lyrae stars, Type II Cepheids, blue stragglers (SX Phoenicis stars), and eclipsing binaries. PILOT will provide a unique data set on these objects, allowing us to put further constraints on the properties of the cluster from analysing large numbers of individual objects.

Finally, by co-adding the time-series photometry we would obtain the deepest and most complete photometric catalogue of $\omega$ Cen, extending down to the white dwarf cooling sequence and the hydrogen burning limit (at $m_{\mathrm{AB}} \approx 27-28$ in $V$ band). A $2 \times 2$ mosaic (four telescope pointings) coverage of the cluster will provide an unprecedented inventory of stars and produce an accurate measurement of the total cluster mass, a crucial parameter for interpreting the history and evolution of $\omega$ Cen.

\subsubsection{Oscillating Stars in Open Clusters}

The southern open clusters NGC 3293 and NGC 4755 have ages around $10 \mathrm{Myr}$ and present a completely different population of stars compared to $\omega$ Cen. Images of the clusters are shown in Figure 4, indicating the fieldof-view of PILOT, while Figure 5 shows the observed colour-magnitude diagrams. In this case, our aim is not to study the cluster history, but rather to improve our understanding of stellar physics. Stellar oscillations are standing sound waves, and a detailed comparison of mode frequencies with stellar evolution models allows rigorous tests of theory. The best studied oscillating star is the Sun, for which helioseismology has allowed measurement of (among other things) the helium content, the depth the convection zone and the internal differential rotation of the Sun.

With PILOT, we propose to apply asteroseismology to stars in these two open clusters. This brings a huge advantage, since we can assume a common age, metallicity and reddening for all the members of the cluster. 

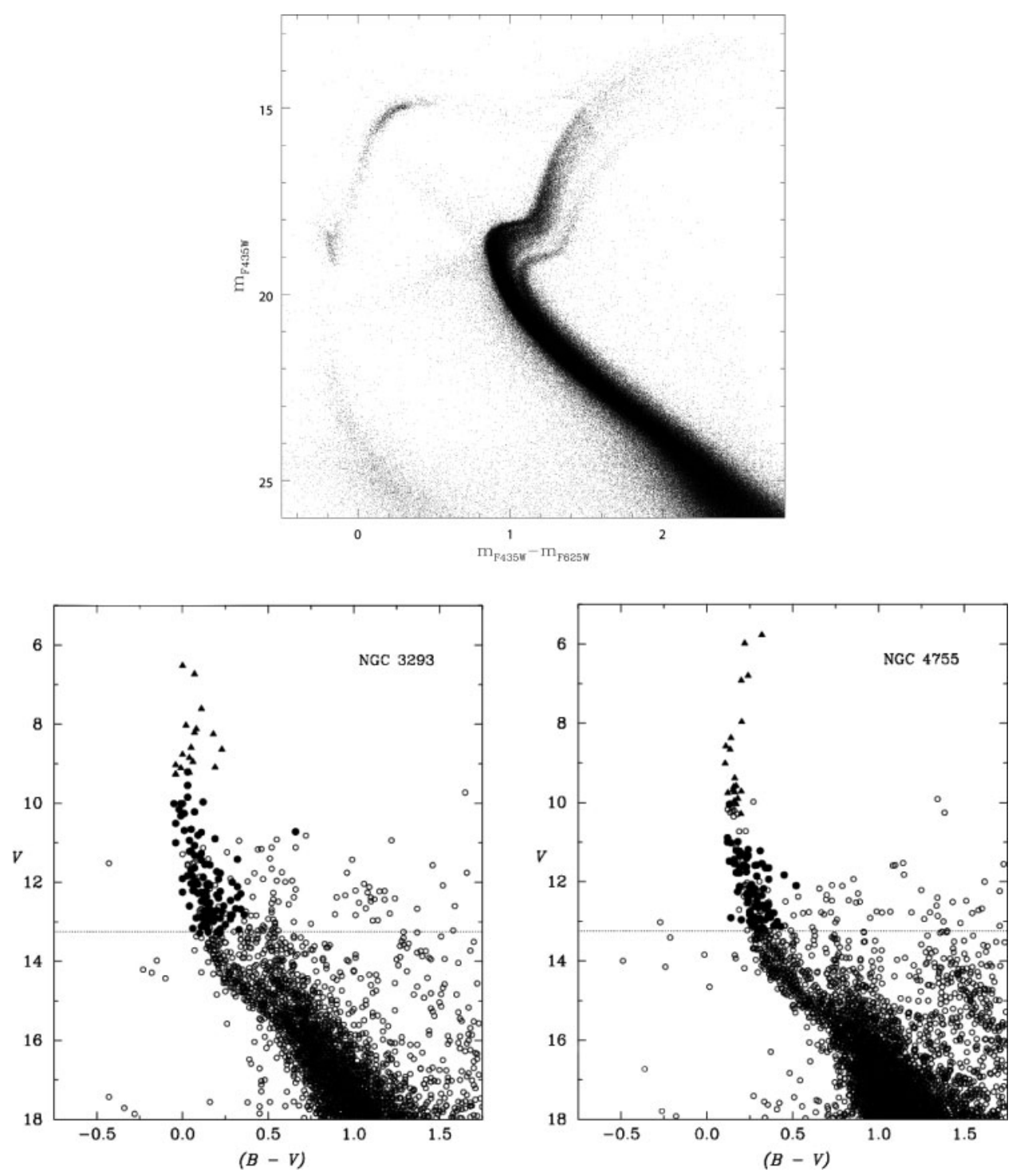

Figure 5 Colour-magnitude diagrams of $\omega$ Cen, NGC 3293 and NGC 4755. Figures from Villanova et al. (2007) and Evans et al. (2005). $\omega$ Cen has an extended horizontal branch and a large white dwarf population. The age of $\omega$ Cen is about 12 Gyr. The open clusters are much younger $(\sim 10 \mathrm{Myr})$ and will allow us to probe B- and A-type stars on the main sequence.

We can therefore study the slight differences from star to star, and in this way make very stringent tests of stellar evolution theory. Especially important are the hot mainsequence stars, some of which oscillate as so-called $\beta$ Cephei variables. Not to be confused with their better known and much cooler cousins, the Cepheid variables, these $\beta$ Cephei stars have masses in the range $4-8 \mathrm{M}_{\odot}$ and oscillate in multiple modes with periods in the range 4-8 hours. Asteroseismology of $\beta$ Cephei stars is an exciting new field that is already providing strong input to theoretical models, particularly regarding the important but poorly-understood processes of rotation and internal mixing (Pigulski 2007). Both clusters are already known to host at least $10 \beta$ Cephei variables, and in NGC 3293, one of them is in a detached eclipsing binary, allowing a direct radius measurement $(P \approx 8 \mathrm{~d}$; Freyhammer et al. 2005). A detailed study of the $\beta$ Cephei stars in these clusters will provide an unrivalled set of oscillation frequencies with which to confront theoretical models and improve our understanding of the physics of massive stars.

This project is likely to require $\sim 2$ weeks of continuous observation per cluster (again, target-of-opportunity breaks are acceptable). Observations will be primarily in the $r$ band. The magnitude range of interest is 8-13, allowing this project to be accomplished in bright sky (twilight/moonlight) conditions.

\section{Star and Planet Formation}

\subsection{Galactic Ecology - Unveiling the Molecular Galaxy}

\subsubsection{Impact}

The site conditions on the summits of the Antarctic plateau makes possible sensitive measurements in the midinfrared of two of the lowest energy lines of molecular hydrogen. This provides an opportunity for PILOT to directly map the principal component of the molecular gas 
in the Galaxy, a project that no other existing or planned facility can undertake.

Because the hydrogen molecule is so light, its rotational energy levels are well spaced, with the lowest lying a few hundred degrees above the ground state. Moreover, these transitions are emitted in a waveband poorly suited for observations from temperate-latitude sites. Hence, the hydrogen molecule has been virtually impossible to use as a probe of the molecular phase, unless in special conditions, such as those that occur in shocks and ultraviolet-excitation, when the near-infrared vibrational lines are excited in gas heated to $\sim 1000 \mathrm{~K}$ (e.g. Burton 1992).

The difficulties associated with observing hydrogen molecules directly has led to the use of trace molecules, such as carbon monoxide, which emit in the millimetre wave bands, as proxies for probing the molecular gas. These lines, however, are generally optically thick, and the abundance and chemical state of the trace molecules is uncertain.

Direct measurement of the ground state molecular hydrogen lines would provide an optically thin probe that samples the entire distribution. Our calculations show that PILOT would be able to directly detect the emission from the $17-\mu \mathrm{m} \mathrm{S}(1)$ and $12-\mu \mathrm{m} \mathrm{S}(2)$ lines of $\mathrm{H}_{2}$ in the typical warm environment of molecular clouds. Moreover, the spatial resolution would be about $2^{\prime \prime}$. This is more than an order of magnitude better than achievable with mapping surveys using millimetre-wave telescopes and is nearly two orders of magnitude better than the current best southern Galactic plane molecular survey, conducted by the NANTEN telescope (Mizuno \& Fukui 2004). The ability to achieve such a step forward in spatial resolution would revolutionise our view of the molecular medium of the Galaxy.

The scientific context here is to understand the life history of the molecular phase of the Galaxy - the processes by which molecular clouds are formed, go on to form stars and are then dispersed. Yet both the formation of molecular clouds and the origin of their physical state remain uncertain. Their masses are much larger than can be supported by thermal pressure, giving rise to the concept of turbulent support. Our picture for the molecular phase, of long-lived entities in quasi-equilibrium, has been giving way to one of a much more dynamic environment, where the clouds and their internal structures may actually be transient features (e.g. Vazquez-Semadeni et al. 2006; Elmegreen 2007). In the new picture, the clouds undergo dynamical evolution, being assembled rapidly by supersonic compressions of the atomic medium, and become self-gravitating in the process. The apparent equilibrium between the turbulent and gravitational pressures then simply arises from the mode of rapid assembly. Star formation can then occur almost as soon as the molecular clouds are formed.

This picture needs to be tested, through a comprehensive examination of the physical environment across the range of spatial and density scales that exist in cloud complexes and their surroundings, across the Galaxy. Central questions are: what is the turbulent energy distribution? Does it relate to the contrasting pictures of local turbulence injection from the natal stellar content (i.e. if virial equilibrium applies) or does it arise from external sources (i.e. if compression applies)? These can be addressed by unveiling the molecular galaxy, i.e. by mapping the distribution of it principal tracer - the hydrogen molecule on the arcsecond scale.

\subsubsection{The Mid-Infrared Lines of Molecular Hydrogen}

Most studies of the molecular hydrogen emission have so far focussed on the near-infrared, where lines from shocks and photodissociation regions are prominent. However, these do not mark the typical state of the molecular gas, only these regions where vigorous activity is occurring, as the emission arises from energy levels several thousand degrees above the ground state.

The lowest rotational lines of the hydrogen molecule, arising from the ground vibrational level and emitted in the mid-infrared, allow the bulk of the gas to be accessed. The ground state line itself, the $28 \mu \mathrm{m} \mathrm{0-0} \mathrm{S(0)} \mathrm{line,} \mathrm{lies} \mathrm{in}$ a region of the spectrum that can only be measured from space, but the $17 \mu \mathrm{m} \mathrm{0-0} \mathrm{S(1)} \mathrm{and} 12 \mu \mathrm{m} \mathrm{0-0} \mathrm{S(2)} \mathrm{lines}$ may be accessed from Antarctica, where the background is an order of magnitude lower than the best temperate sites, and the sky stability permits staring observations.

The bulk of the molecular gas is not at the $\sim 10-20 \mathrm{~K}$ commonly associated with cold $\mathrm{CO}$ emitting molecular clouds, but rather is at temperatures of order $\sim 100 \mathrm{~K}$. Much of this exists in a photodissociation region (PDR) environment, warmed by the ambient far-ultraviolet radiation fields to this temperature. These are the surface layers of molecular clouds, but in fact comprise much of the molecular environment, outside the cold, dense cores within the clouds where star formation is initiated. Furthermore, it has also become clear through observations made with the ISO satellite that intensities in the mid-infrared lines are significantly higher in many instances than would be expected from simply PDR-excited molecular clouds (e.g. Habart et al. 2005).

Extensive regions of warm molecular gas $(T>100 \mathrm{~K})$ exist throughout the cold interstellar medium, for which ultraviolet photons cannot be the sole heating source. This has led to the conjecture that turbulent heating may be significant. Turbulent injection of energy and its dissipation is suspected of being the primary agent responsible for the initiation and regulation of star formation through molecular clouds (e.g. Falgarone et al. 2005). However, direct evidence for this is lacking because of inability to probe the relevant molecular environment where this would occur. The warm, molecular gas traced by the midinfrared lines of molecular hydrogen are needed for this task, as they are sensitive to the emission from weak shocks (e.g. $V_{\text {shock }} \approx 5 \mathrm{~km} \mathrm{~s}^{-1}$ ) where dissipation might occur.

There are even suggestions that in several external galaxies some baryonic dark matter may be hidden inside such molecular gas, and has remained undetectable to date 
(e.g. Pfenniger, Combers \& Martinet 1994). The ability to trace the molecular medium in its primary constituent, rather than in a trace one, would allow more reliable estimates to be made of its contribution to the total baryonic mass content, and so assess the importance to the dark matter budget.

The mid-infrared $\mathrm{H}_{2}$ lines will also be sensitive to regions of activity in the molecular gas, for instance where it is heated by shock waves from outflows from young stellar objects, or by fluorescent emission from ultraviolet photons generated by young stars nearby (e.g. Burton, Hollenbach \& Tielens 1992). These are generally much brighter than the thermal emission from the quiescent gas, and so will be readily detected. Hence, the proposed surveys will also be sensitive to the most active regions of molecular gas across the Galaxy. While a dedicated survey for such active regions alone might be better carried out through imaging the vibrational-rotational lines of $\mathrm{H}_{2}$ in the $2 \mu \mathrm{m}$ band, this information will be obtained at no extra cost through the survey we propose here.

\subsubsection{Other Facilities}

While the molecular hydrogen ground vibrational state $\mathrm{S}(1)$ and $\mathrm{S}$ (2) lines can be observed from excellent observing sites such as Mauna Kea, there has been limited work undertaken in this arena. The high background and unstable observing conditions preclude extensive surveys, and there have been none proposed for such temperate sites. Pointed, spectroscopic observation of a few bright sources is all that has been done. Airborne observations, such as from SOFIA, might also access the $\mathrm{S}(1)$ and $\mathrm{S}(2)$ lines (but not the $28 \mu \mathrm{m} \mathrm{S}(0)$ line); the only instruments proposed, however, are spectrometers, able to obtain complete spectra across the relevant wavebands. It would not be possible to conduct an imaging survey with the limited amount of observing time that would be available from SOFIA.

Observations from space, with cryogenic instrumentation, are, of course, vastly more sensitive than from even the best ground-based observatories operating in the thermal infrared. However, the space observatories concentrate on deep photometric imaging in the infrared bands, together with low spectral resolution spectroscopic capabilities for pointed observations. For instance, JWST will be able to take spectra of the mid-infrared $\mathrm{H}_{2}$ lines in selected sources, but will not conduct imaging surveys of them. A proposal for a suitable $\mathrm{H}_{2}$ line imager, $\mathrm{H} 2 \mathrm{EX}$ (Boulanger et al. 2008), was submitted to the ESA Cosmic Visions program, but was not selected for further study.

There is thus no competition for PILOT for widefield, spectroscopic, mid-infrared line imaging surveys from any other facility. This provides a unique opportunity for the telescope, should there also be scientifically worthy projects to conduct. One such project clearly stands out: imaging the molecular gas of our Galaxy in its primary component. Such a facility would revolutionise our view of the molecular medium of the Galaxy, and our understanding of the Galactic ecology.

\subsubsection{Observations}

We envisage a camera containing a wide-field FabryPerot, tuneable to the wavelengths of interest, used in conjunction with a large format, mid-infrared array detector - this is PMIRIS. With several months of dedicated telescope time, which could be in daylight as this makes little difference in Antarctica, images of the molecular hydrogen emission could be obtained across extensive regions of the Southern Galactic Plane.

PILOT would achieve a $1 \sigma$ sensitivity in 10 minutes to the $17 \mu \mathrm{m} \mathrm{S}(1)$ and $12 \mu \mathrm{m} \mathrm{S}(2)$ lines of $2 \times 10^{-5}$ and $3 \times 10^{-6} \mathrm{erg} \mathrm{cm}^{-2} \mathrm{~s}^{-1} \mathrm{sr}^{-1}$, respectively ${ }^{5}$. These calculations assume background limited operation, with background fluxes of 1000 and $30 \mathrm{Jy} / \mathrm{arcsec}^{2}$ and atmospheric transmissions of 0.7 and 0.98 , at wavelengths of $17 \mu \mathrm{m}$ and $12 \mu \mathrm{m}$, respectively (the conditions are particularly favourable in Antarctica for observation of the $12 \mu \mathrm{m}$ line). They assume an instrumental efficiency of 0.3 , a spectral resolution of $15000\left(\Delta V=20 \mathrm{~km} \mathrm{~s}^{-1}\right)$, and $2^{\prime \prime}$ pixel size (matched to the diffraction limit for the $17 \mu \mathrm{m}$ $\mathrm{S}(1)$ line). For well depths of $10^{6}$ electrons for the detectors, read out would need to be every $\sim 1$ second and $\sim 1$ minute, for the two lines respectively; i.e. it would not be necessary to chop, but staring observations would suffice.

For a column density of molecular gas equivalent to an optical depth of unity (i.e. $A_{V} \approx 1 \equiv \mathrm{NH}_{2}=10^{21} \mathrm{~cm}^{-2}$ ), these sensitivities equate to being able to detect molecular gas warmer than $\sim 140 \mathrm{~K}$ and $\sim 165 \mathrm{~K}$ for the $\mathrm{S}(1)$ and $\mathrm{S}(2)$ lines, respectively ${ }^{6}$ - see Figure 6 . Typical photodissociation regions have columns of $A_{v} \approx 3-4$ for their warm surface layers, so these sensitivities should be sufficient to pick up gas to these limits in such environments.

These sensitivities should be compared to measurements already made of the lines to demonstrate they are sufficient for the science needs. For instance, the Orion molecular cloud shock exhibits line intensities of $\sim 5 \times 10^{-3} \mathrm{erg} \mathrm{cm}^{-2} \mathrm{~s}^{-1} \mathrm{sr}^{-1}$, when seen through $\sim 20^{\prime \prime}$ beams (Parmar, Lacy \& Achtermann 1994; Burton \& Haas 1997), and while the $\mathrm{H}_{2}$ emission in this source is an order of magnitude brighter than the typical Galactic source that is studied, these fluxes are still another order of magnitude brighter than the sensitivity limit. Similarly, the photodissociation region in Orion has mid-infrared $\mathrm{H}_{2}$ line intensities of $\sim 3 \times 10^{-4} \mathrm{erg} \mathrm{cm}^{-2} \mathrm{~s}^{-1} \mathrm{sr}^{-1}$, an order of magnitude above the sensitivity level (Parmar, Lacy \& Achtermann 1991). Over the Central Molecular Zone, the ISO satellite

\footnotetext{
${ }^{5}$ Note that, while the $28 \mu \mathrm{m} \mathrm{0-0} \mathrm{S(0)} \mathrm{line} \mathrm{is} \mathrm{unobservable} \mathrm{from} \mathrm{any}$ Earth-based location, the $9.6 \mu \mathrm{m} 0-0 \mathrm{~S}(3)$ is also poorly situated for observation, lying in a strong atmospheric ozone feature.

${ }^{6}$ For comparison, 1 minute integrations achieve sensitivities and equivalent temperatures of $6 \times 10^{-5} \mathrm{erg} \mathrm{cm}^{-2} \mathrm{~s}^{-1} \mathrm{sr}^{-1} \& 170 \mathrm{~K}$ and $10^{-5} \mathrm{erg} \mathrm{cm}^{-2} \mathrm{~s}^{-1} \mathrm{sr}^{-1} \& 190 \mathrm{~K}$, respectively for the $\mathrm{S}(1)$ and $\mathrm{S}(2)$ lines. 1 hour integrations will achieve $8 \times 10^{-6} \mathrm{erg} \mathrm{cm}^{-2} \mathrm{~s}^{-1} \mathrm{sr}^{-1} \& 120 \mathrm{~K}$ and $10^{-6} \mathrm{erg} \mathrm{cm}^{-2} \mathrm{~s}^{-1} \mathrm{sr}^{-1} \& 150 \mathrm{~K}$. Note the cooler temperatures reached by using the $\mathrm{S}(1)$ line, despite the poorer sensitivity at $17 \mu \mathrm{m}$. This is because the upper energy level is lower than that of the $S(2)$ line.
} 


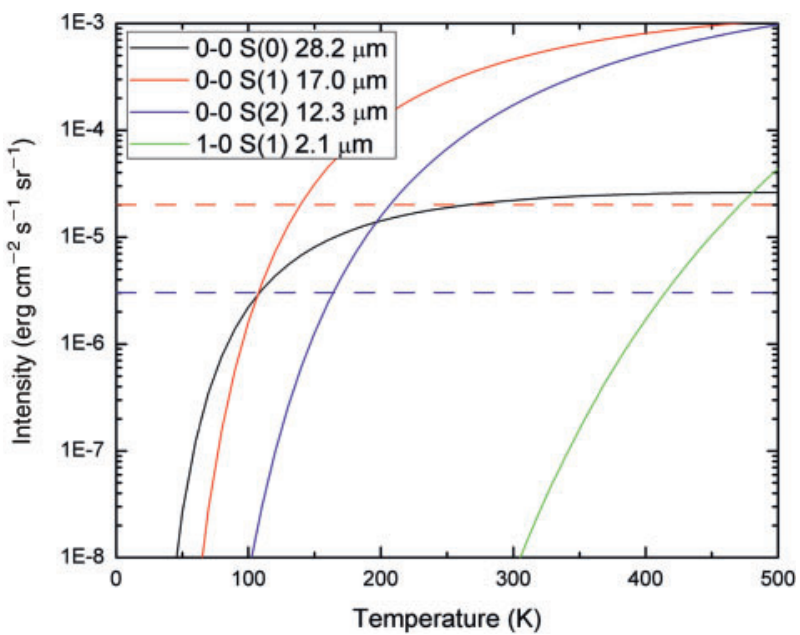

Figure 6 Intensities of the molecular hydrogen emission lines from the mid-infrared $0-0 \mathrm{~S}(0), \mathrm{S}(1)$ and $\mathrm{S}(2)$ lines $(28.2,17.0$, $12.3 \mu \mathrm{m})$, as well as the commonly observed near-infrared 1-0 S(1) line $(2.12 \mu \mathrm{m})$, as a function of the gas temperature. These are for a column density of gas equivalent to a unit optical depth (i.e. $\mathrm{NH}_{2} \approx 10^{21} \mathrm{~cm}^{2}$ ), typical of the surface layer for a molecular cloud, and assume LTE. The horizontal lines show the $1 \sigma$ intensities that would be obtained in 10 minutes integration time with PMIRIS for the $S(1)$ and $S(2)$ lines. As is readily apparent, the near-infrared 1-0 $\mathrm{S}(1)$ line is unobservable in warm molecular gas. While the $0-0 \mathrm{~S}(0)$ line is the most sensitive to warm molecular gas, down to $\sim 100 \mathrm{~K}$, it is unobservable from the ground. The 0-0 S(1) and $S(2)$ lines therefore provide the most ready access for directly sampling the principal component of molecular clouds. Note that, while the sensitivity to the $\mathrm{S}(2)$ line is greater, its higher energy level $(1682 \mathrm{~K}$ cf. $1015 \mathrm{~K}$ for the $S(1)$ line) means that the $S(1)$ line is sensitive to cooler gas. It is emitted at a wavelength where the Antarctic site is greatly superior to temperate-latitude observatories.

measured intensities of $\sim 10^{-4} \mathrm{erg} \mathrm{cm}^{-2} \mathrm{~s}^{-1} \mathrm{sr}^{-1}$ over large $\left(14^{\prime \prime} \times 27^{\prime \prime}\right)$ beams (Rodríguez-Fernández et al. 2001) at a few selected positions. Even if the emission is uniformly distributed over these beams, it would still be readily measurable with the survey parameters above. Model predictions for a variety of molecular shock types show that the mid-infrared lines will be readily detectable at the achievable sensitivity levels, when the emission fills the pixel (Burton et al. 1992). They will also be detectable in the photodissociation region environment when the ambient far-ultraviolet radiation field, $G_{0}$, is $10^{4}$ or more times higher than the average interstellar radiation field as occurs when there is a nearby OB star.

The velocity spread of molecular hydrogen line emission across a typical Galactic source would be no more than $\sim 150 \mathrm{~km} \mathrm{~s}^{-1}$. In the Central Molecular Zone, however, the emission is spread over $\sim 250 \mathrm{~km} \mathrm{~s}^{-1}$. The wavelength change experienced by rays passing through the plates at different angles when using a Fabry-Perot etalon, provides a means of also measuring these motions. In order to image all the line emission across the field-of-view of the detector, the plate separation needs to be scanned over the complete wavelength range across the etalon. Alternatively, the etalon can be kept with a fixed spacing, and the telescope moved in small steps $\left(\sim 1^{\prime}\right)$ across the array, so that each spatial position is observed at many different phases across the complete wavelength range. Since the observations would be background limited, a gain in sensitivity is achieved by using higher spectral resolution (so long as the line is not resolved). Thus, the use of a Fabry-Perot permits dynamical information on the source to be obtained in addition to the imaging — through constructing a spatial-velocity cube of the line emission.

As an example of a possible survey that could be conducted, we consider a circular etalon of diameter $15 \mathrm{~cm}$, imaging over an array where the square aperture contained within the etalon is $10^{\prime}$ on a side. Using a fixed etalon spacing, taking 1 minute of integration per position, and stepping the telescope along a $1^{\prime}$ grid, each position in space would be imaged 100 times, covering the phase velocity range of $175 \mathrm{~km} \mathrm{~s}^{-1}$. For a Fabry-Perot with spectral resolution $20 \mathrm{~km} \mathrm{~s}^{-1}$, this yields $\sim 10$ minutes of integration per spectral resolution element per position (the precise time depends on the velocity with respect to that at the phase centre). To map a degree-sized field typical of a giant molecular cloud would then require $\sim 10$ days of observing (assuming a $25 \%$ duty cycle). To map the complete $3^{\circ} \times 1^{\circ}$ area of the Central Molecular Zone, which would also require two separated spacings of the etalon plates in order to cover the $\sim 250 \mathrm{~km} \mathrm{~s}^{-1}$ wide extent of the emission, would require $\sim 60$ days observing. In either case, the observations would be sensitive to the presence of molecular gas warmer than $\sim 140 \mathrm{~K}$. Such a project could be accomplished over a single summer observing period.

In addition to $\mathrm{H}_{2}$ there are many other spectral lines in the mid-infrared bands that could also be imaged with a Fabry-Perot instrument. These are mostly fine structure atomic lines. Some are particularly strong coolants of the interstellar medium, for instance the [NeII] $12.8 \mu \mathrm{m}$ line, a dominant line emitted from HII regions. At $12.8 \mu \mathrm{m}$ the emission is nearly extinction free, so an imaging survey in the [NeII] line would provide an unbiased view of HII regions across the Galaxy, providing dynamical as well as spatial information. Such a survey could be conducted very much more quickly than the molecular hydrogen imaging survey.

\subsection{Disc Populations in the Chamaeleon Dark Clouds Complex}

The studies of circumstellar discs specifically around young low-mass stellar objects (YSOs) and young brown dwarfs (BDs) offer an opportunity to explore planet formation. The primordial circumstellar discs around low-mass stars and brown dwarfs can be easily detected through observations at mid-infrared wavelengths longer than $5-10 \mu \mathrm{m}$ in which there is the best combination of contrast of the disc relative to the stellar photosphere. In addition, measuring the lowest mass at which young objects harbour circumstellar discs is crucial for determining whether planets can form around low-mass BDs. In the last few years, thanks to the infrared satellites ISO and Spitzer, much progress has been made in this subject. Using the IRAC and MIPS cameras on the Spitzer telescope, large 
13h $14 \quad 12 h 57 \quad 12 h 38 \quad 12 h 19 \quad 11 h 59 \quad 11 h 40 \quad 11 h 21 \quad 11 h 02$ 10h44

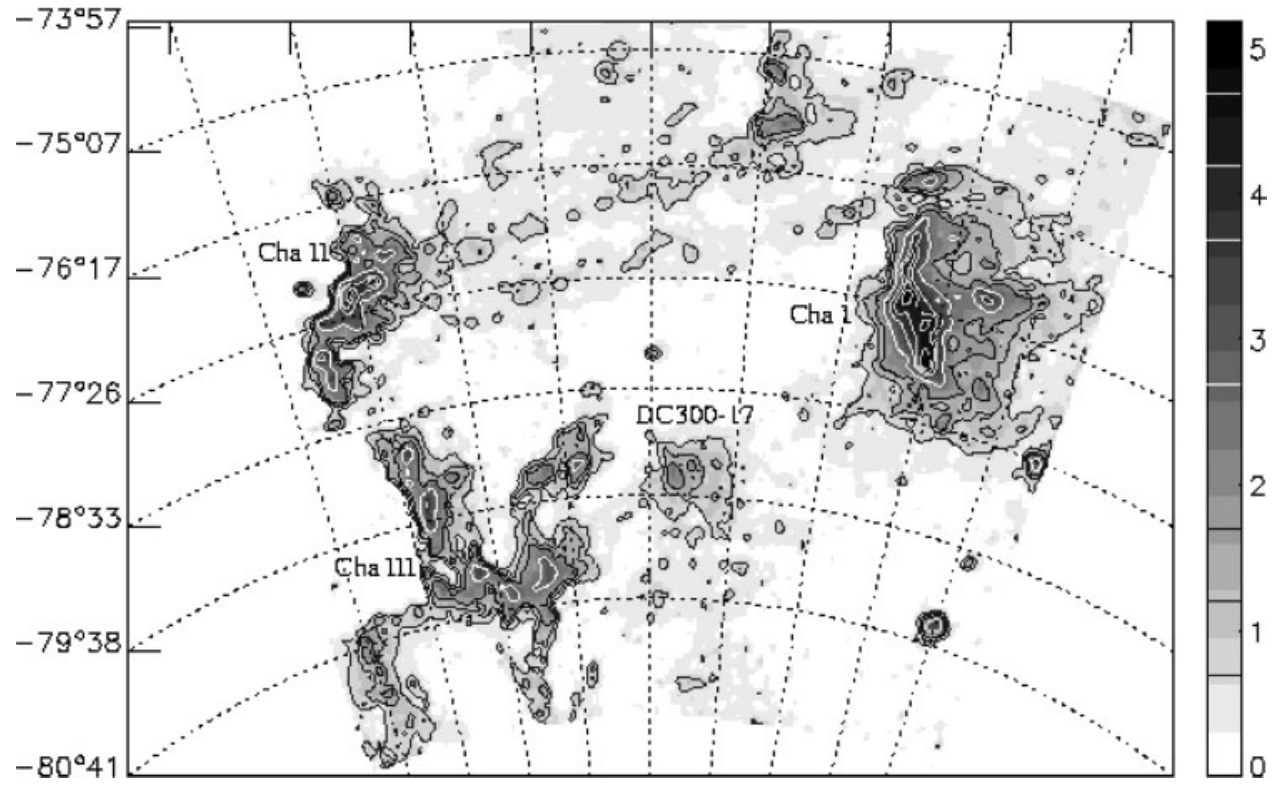

Figure 7 Visual extinction map of the Chamaeleon dark clouds complex. From Cambrèsy (1999).

surveys of nearby star forming regions and young associations, such as Taurus, Perseus, Lupus, Serpens, and Chamaeleon (e.g. see Luhman et al. 2008a, and references therein), have identified disc-bearing members at different masses and stages of evolution.

The southern constellation of Chamaeleon (at a distance of $\sim 160-180 \mathrm{pc}$ ) contains one of the nearest groups of dark clouds to the Sun. This complex, shown in Figure 7, is formed of at least six clouds as observed from the large scale ${ }^{12} \mathrm{CO}(\mathrm{J}=1-0)$ map of Mizuno et al. (2001) and the $A_{V}$ map of Cambrèsy (1999). Chamaeleon was also the subject of investigation using the SPIREX South Pole telescope (Kenyon \& Gómez 2006); 58\% of 124 sources detected in Cha I at $L$ band showed near-infrared excess emission characteristics of discs. Only small regions of the three main clouds (Cha I, Cha II, and Cha III) have been extensively observed in the mid-infrared with ISOCAM, the infrared camera of the ISO satellite (Persi et al. 2000, 2003), and with Spitzer. The results of these surveys are well summarized by Luhman et al. (2008b). In an area of a few square degrees of Cha I, 237 low-mass YSOs have been found, of which 33 have been identified as young BDs. Approximately $50 \%$ of these sources with masses from 0.01 to $0.3 \mathrm{M}_{\odot}$ have discs. One of the most exciting results is the discovery by Luhman et al. (2005) of a circumstellar disc in one of the less massive young BDs $\left(M \approx 8 M_{J}\right)$. About 50 members of Cha II have been identified, including a few brown dwarfs (Alcalà et al. 2008). In Cha III, no sources with infrared excess have been found (Persi, Marenzi \& Gómez 2002), suggesting that the cloud is probably at an early evolutionary stage of the star forming complex.

The ISOCAM and Spitzer surveys of this region cover only a small fraction $(5-8 \%)$ of the total area ( $\sim 64 \mathrm{deg}^{2}$, see Figure 7$)$. In addition, one of the limitations represented by these surveys in the mid-infrared is the poor spatial resolution ( $\sim 6^{\prime \prime}$ FWHM at $24 \mu \mathrm{m}$ for Spitzer). This prevents the detection of multiple systems associated with YSOs and young BDs, which are required to obtain a correct census of the young stellar population in Chamaeleon that is fundamental to derive its initial mass function (IMF). Figure 8 shows, as an example, one of these multiple systems found in Cha I by Persi et al. (2001). The two YSOs have been classified as Class I sources from their spectral energy distribution and are not separated in the Spitzer and ISOCAM images.

The project proposed here for PILOT includes, as a first step, the survey of the whole $\left(8^{\circ} \times 8^{\circ}\right)$ Chamaeleon dark clouds complex in three different filters between 8 and $21 \mu \mathrm{m}$. The required spatial resolution scales with the diffraction limit of the PILOT telescope from 1" (FWHM) at $\sim 10 \mu \mathrm{m}$ to $1.8^{\prime \prime}$ at $20 \mu \mathrm{m}$; this is a factor 3 better than the MIPS resolution at $24 \mu \mathrm{m}$. This will allow a complete census of YSOs and young BDs from the analysis of the derived mid-infrared colour-colour plot. The luminosities of these objects will be derived using $J$ and $H$ magnitudes obtained from the 2MASS and VISTA surveys. It will thus be possible to obtain the most complete IMF for this complex.

These observations can also be made using existing large telescopes (e.g. Gemini South, VLT) equipped with mid-infrared imaging spectrometers (e.g. Michelle). However, such facilities cannot be used to carry out large-scale mid-infrared surveys at reasonable spatial resolution proposed here, because of their small field-of-view and the instability of the $20 \mu \mathrm{m}$ window at mid-latitude locations. Current and near-future infrared space missions such as AKARI and WISE will cover these wavelengths, but have a poor spatial resolution and are thus not suitable to develop the proposed project. 

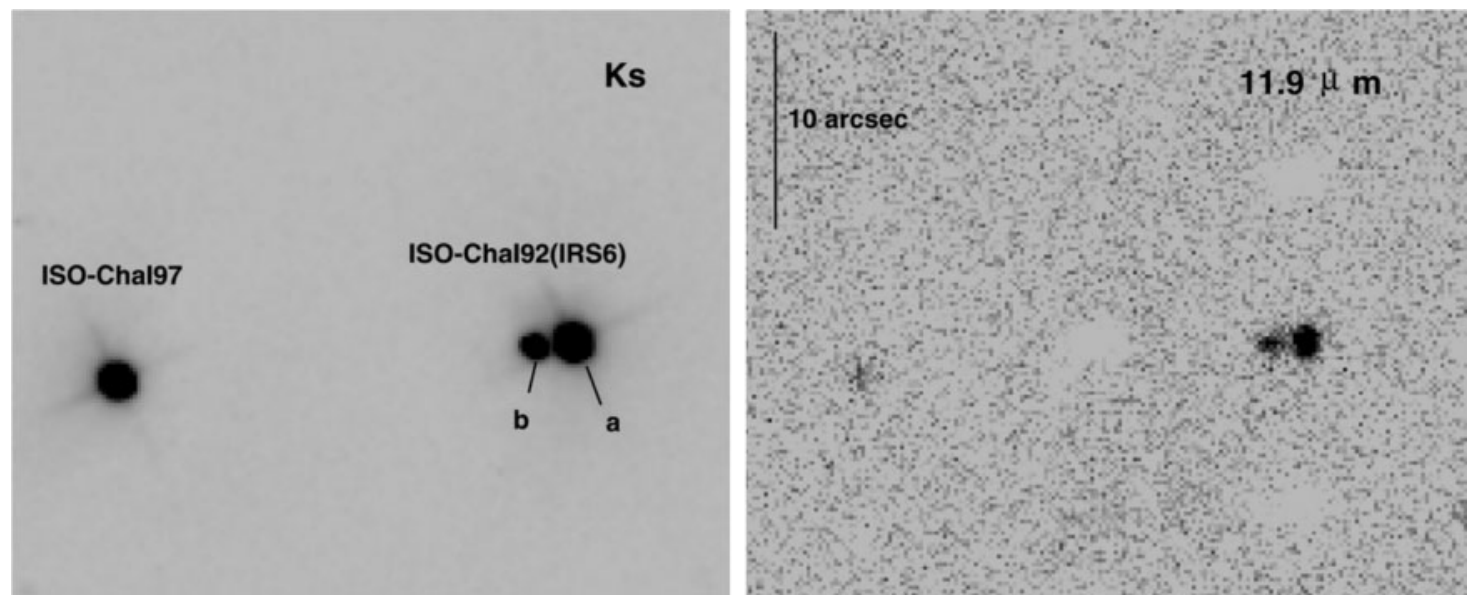

Figure $8 K_{\mathrm{s}}$-band (left) and 11.9- $\mu \mathrm{m}$ (right) images, obtained with the ESO $3.6 \mathrm{~m}$ New Technology Telescope, of the binary system ISO-ChaI92 composed of two Class I YSOs. Adapted from Persi et al. (2001).

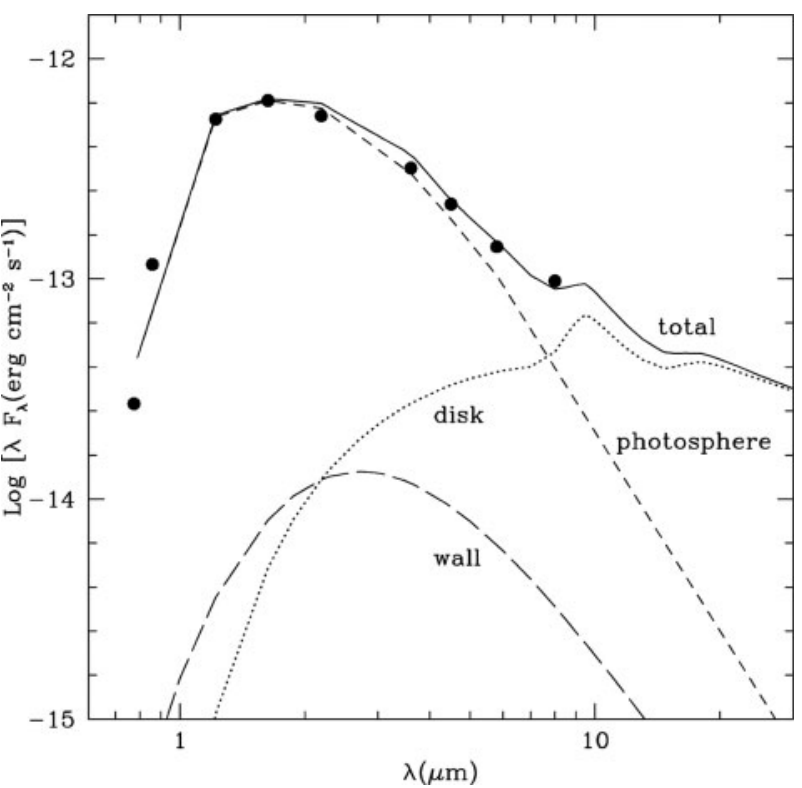

Figure 9 Spectral energy distribution of the young brown dwarf Cha1 109-7734 with mass $\sim 8 M_{J}$. The observed excess flux at wavelengths greater than $5 \mu \mathrm{m}$ is modelled in terms of emission from a circumstellar accretion disc. From Luhman et al. (2005).

As a second step of this project, photometry at longer wavelengths $(30-40 \mu \mathrm{m})$ of the discovered YSOs and young BDs is required. This, together with the nearinfrared and the mid-infrared flux densities obtained in the survey, will allow spectral energy distributions (i.e. as shown in Figure 9) to be built up over a wide range of wavelengths. From comparison with existing models (e.g. Robitaille et al. 2007), it will be possible to derive the physical characteristics of the circumstellar discs.

The baseline mid-infrared PILOT camera, PMIRIS, is suitable for this project. The large survey will be made using the blue channel of this instrument with filters, chosen to have a broad wavelength coverage, centred at 8.9, 12.7 , and $21 \mu \mathrm{m}$. With a $14^{\prime} \times 14^{\prime}$ field-of-view, $\sim 1400$ frames per filter are necessary to survey the total area of the Chamaeleon dark clouds, including an overlapping of
$20 \%$. The survey depth should be on the order of $5 \mathrm{mJy}$ at $21 \mu \mathrm{m}$, based on MIPS observations of Cha I and Cha II. This represents an integration of $\sim 1$ hour per frame at the PILOT $R=20$ sensitivity, or $\sim 1400$ hours total for the $21 \mu \mathrm{m}$ survey. Less time is required to obtain a similar depth at 8.9 and $12.7 \mu \mathrm{m}$. The total observing time for three filters is thus about 3000 hours. An extra time of $\sim 500$ hours will be necessary for photometry in one broad-band filter in the $30-40 \mu \mathrm{m}$ region using the red arm of PMIRIS for observations of young low flux YSOs and young BDs.

\subsection{Crystalline Silicates}

Dust grains in high temperature environments (e.g. protoplanetary discs and planetary nebulae) undergo thermal processes which can be traced by the fraction of crystalline silicates. The growth and crystallisation of sub-micron sized dust grains marks the onset of planet formation in protoplanetary discs. Magnesium-rich compounds (forsterite, enstatite) have spectral signatures in the mid-infrared, from 7 to $40 \mu \mathrm{m}$.

These features have been found in the $7-14 \mu \mathrm{m}$ wavelength range by Apai et al. (2005) in a sample of young BDs in Cha I, as shown in Figure 10. However, it is difficult to disentangle the contributions from the organic molecules (PAHs), crystalline silicates, and amorphous silicates in this wavelength range. Features at $\sim 28 \mu \mathrm{m}$ and $\sim 34 \mu \mathrm{m}$ are only produced by crystalline silicates (see Molster \& Kemper 2005). Figure 11 shows this spectral range for several $\mathrm{T}$ Tauri and Herbig Ae/Be stars. PILOT could study $\mathrm{Mg}$ compounds in nearby $(\sim 200 \mathrm{pc})$ bright protoplanetary discs around BDs and very low-mass YSOs.

While ISO/SWS and Spitzer/IRS have provided a small sample of low resolution spectra of circumstellar discs in the wavelength range $25-40 \mu \mathrm{m}$ (e.g. Morrow et al. 2008), no systematic survey has been undertaken or is currently foreseen. No existing or planned ground-based telescope is sensitive in this wavelength range. The high 


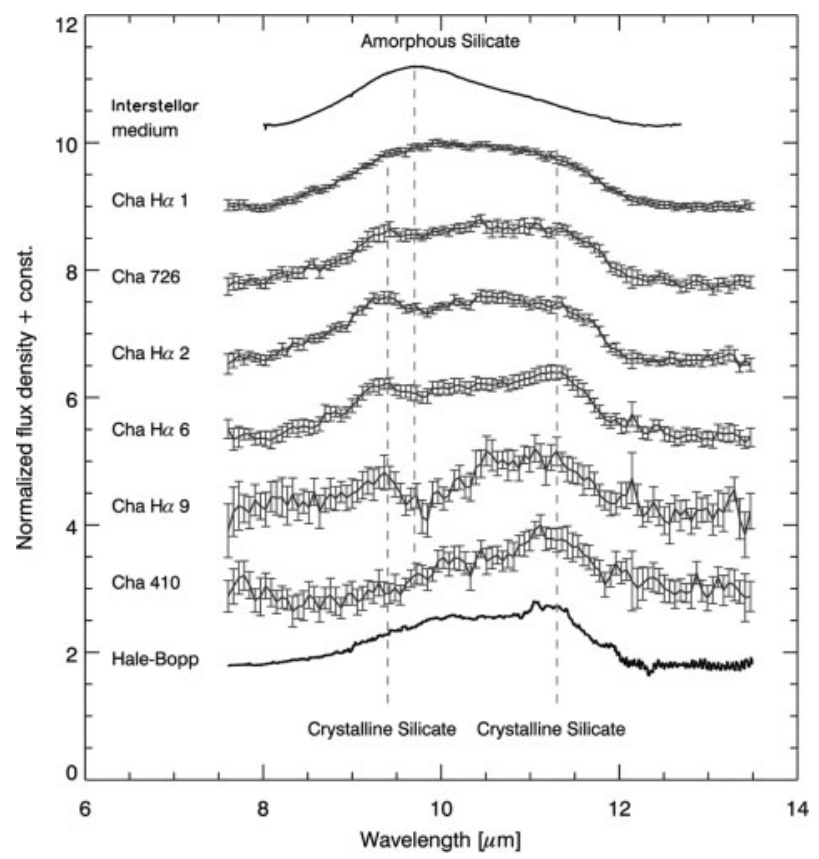

Figure 10 Mid-infrared spectra of young BDs in Cha I obtained with the Spitzer Infrared Spectrometer, compared with the spectra of the amorphous silicate-dominated interstellar medium, and the crystalline-rich comet Hale-Bopp. From Apai et al. (2005).

wavelength cut-off for the MIRI instrument ${ }^{7}$ on JWST is $27-29 \mu \mathrm{m}$, the highest filter wavelength on WISE is $23 \mu \mathrm{m}$, and the AKARI satellite only observes shortward and longward of this range. The only future facility sensitive in this region of the mid-infrared is the FORCAST instrument on SOFIA (Adams et al. 2006). However, as one of a complement of 11 planned first generation instruments ${ }^{8}$, observing time constraints will likely prevent it from performing large-area spectroscopic surveys. The spectroscopic characterisation of a large sample of circumstellar discs, required to make breakthrough advances in this field, can thus only be achieved with the PILOT telescope.

The project proposed here for PILOT is to perform a survey of the brightest nearby protoplanetary discs in the $17-40 \mu \mathrm{m}$ wavelength range. Such a survey would allow the thermal evolution of circumstellar discs to be traced, from the embedded to the pre-main sequence and debris phases. This is a unique science case, because there are no other facilities capable of performing such a systematic survey for $\mathrm{Mg}$ silicates in this wavelength range.

This science case requires targeted low resolution $(R \approx 500)$ grism spectroscopic observations in the range $17-40 \mu \mathrm{m}$. This could be accomplished with the red arm of the PMIRIS instrument. The estimated observing limit is for a bright protoplanetary disc at up to $\sim 200 \mathrm{pc}$ for 1 hour of observing time ( $m_{\mathrm{AB}} \approx 9$ at $\left.30 \mu \mathrm{m}\right)$. No significant temporal variability is expected, so the objects would only be

\footnotetext{
${ }^{7}$ See http: //www. jwst. nasa.gov/miri.html.

${ }^{8}$ Seehttp://www.sofia.usra.edu/Science/instruments/ sci_instruments.html.
}

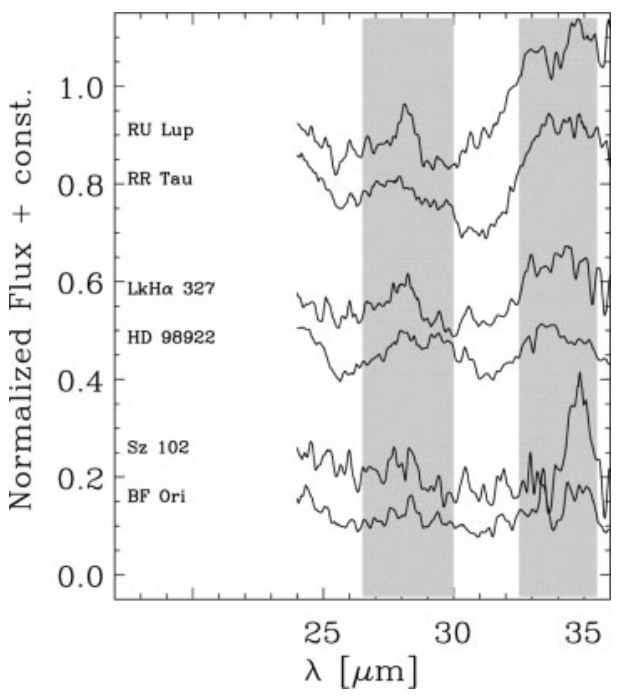

Figure 11 A selection of spectra, after subtraction of disc emission, from sources showing emission from crystalline silicates in the $\sim 28$ and $\sim 34 \mu \mathrm{m}$ (shaded) regions. Each pair of spectra contains one Herbig Ae/Be star (upper) and one T Tauri star (lower). A high fraction of pre-main sequence stars ( $\sim 50 \%$ for $\mathrm{T}$ Tauri stars) are found to exhibit such emission features. From Kessler-Silacci et al. (2006).

observed once. The full characterisation of all circumstellar discs bright enough to be observable from Dome $\mathrm{C}$ is a long-term project that will probably need more than one observing season. It is likely that the brightest objects in the close Chamaeleon star forming region could be completely observed during a single year, and the previously proposed Chamaeleon dark clouds survey for circumstellar discs would provide the obvious source catalogue for such a project.

\subsection{Embedded Young Stellar Objects}

Class 0/I Young Stellar Objects (YSOs, protostars surrounded by extended envelopes) are cool objects ( $\sim 30$ $70 \mathrm{~K})$. The emission maximum occurs at $\sim 40-100 \mu \mathrm{m}$. Therefore, they can be best studied in the mid- to farinfrared, and space-based missions (IRAS, ISO, Spitzer) have been essential to characterise and model these objects. Mid-infrared fluxes can effectively constrain YSO models (see for example Figure 12) and thus be used to estimate physical parameters such as stellar temperature, disc mass, and accretion rate (Robitaille et al. 2006, 2007). Both high sensitivity and high spatial resolution are required to break the many model degeneracies.

The space mission WISE is going to provide a global survey from 3.3 to $23 \mu \mathrm{m}$ with unprecedented sensitivity but low spatial resolution. WISE will discover many embedded YSOs and unresolved mid-infrared sources, but will not be able to fully characterise them (Mainzer et al. 2006). PILOT could make follow-up observations in the spectral range $17-40 \mu \mathrm{m}$ with moderate spatial resolution, and provide key insight into the study of YSOs.

The specific outcome of the proposed study is to obtain a photometric characterisation of all the embedded YSOs 

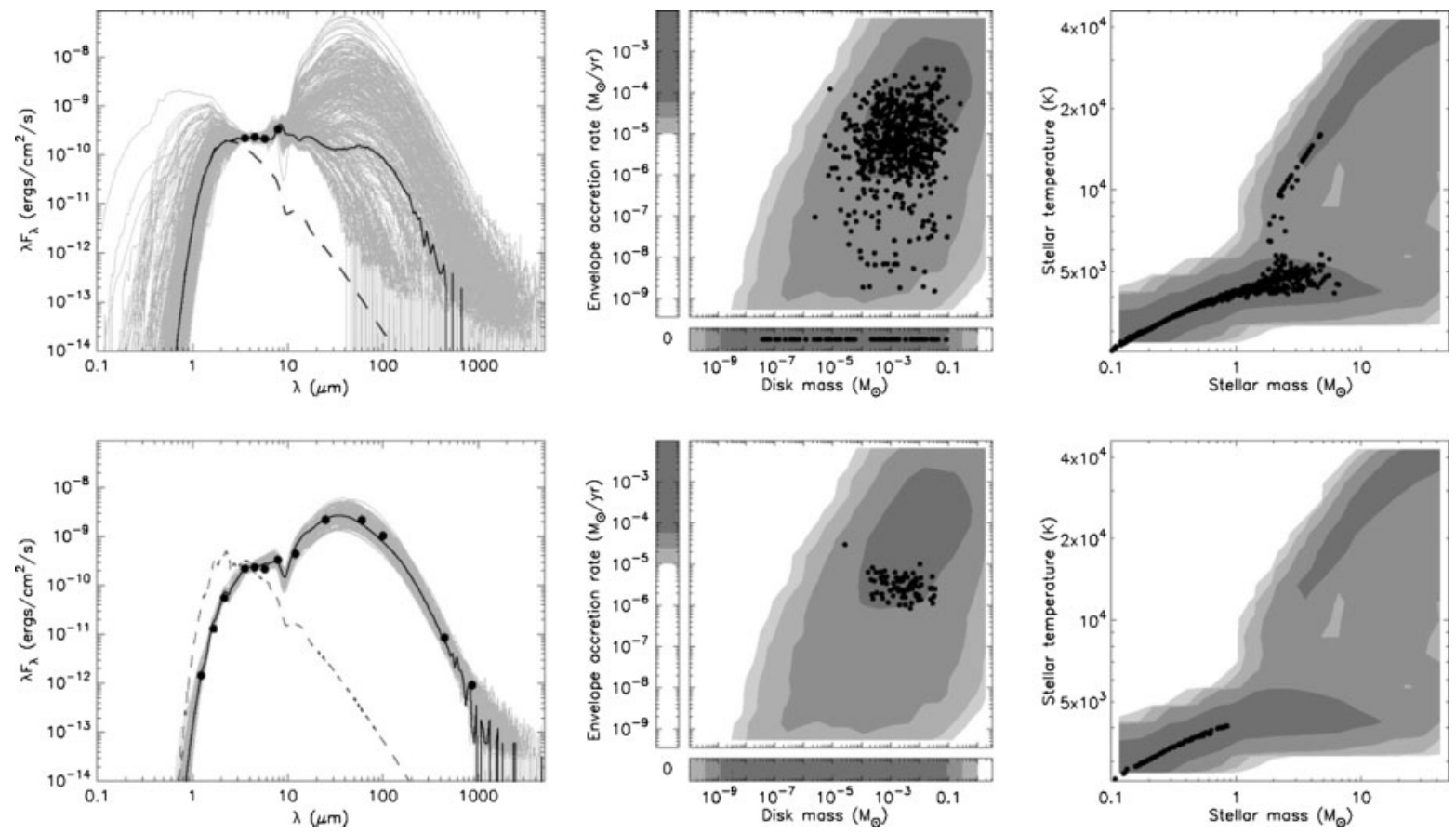

Figure 12 The embedded object IRAS 04361+2547 spectral energy distribution (left column) has been modelled using an increasing number of data points over a wider wavelength range (top to bottom row). The mid- to far-infrared data effectively constrains the physical properties of the object (centre and right columns). Adapted from Robitaille et al. (2007).

observable from Dome $\mathrm{C}$ in the $17-40 \mu \mathrm{m}$ range, and to obtain low resolution spectroscopy of the brightest objects in the same interval. This project will enable star formation to be studied in the less known very early stages, allowing important new insights to be gained for embedded young stellar objects. Embedded YSOs could be first studied in a systematic way in the wavelength range where many of them have their maximum emission peaks, and ground-based temperate-location observatories have zero atmospheric transmission.

Similar to the case for crystalline silicate observation, Spitzer/IRS has provided a small sample of embedded sources with low resolution spectra in the wavelength range $25-40 \mu \mathrm{m}$. No systematic photometric survey has been undertaken or is currently foreseen. PILOT is the only facility (space or ground-based) apart from SOFIA/FORECAST that will be capable of observing in this wavelength range.

The primary observing mode for this project is pointed observations of previously discovered WISE embedded YSOs with the red arm of the PMIRIS instrument on PILOT. Additionally, small surveys of molecular cloud cores may be undertaken. The estimated observing limit is a solar mass embedded object at $5 \mathrm{kpc}$ for 1 hour of observing time $\left(m_{\mathrm{AB}}=12.2\right.$ at $\left.30 \mu \mathrm{m}\right)$. No temporal variability is expected, so the fields would only be observed once.

Ideally, five filters would be used to accurately trace the spectral energy distribution over the range $17-40 \mu \mathrm{m}$. The full characterisation of all embedded protostars observable from Dome $\mathrm{C}$ will need more than one observing season. Nearby star forming regions (e.g. Chamaeleon, Lupus) could be completely photometrically characterized during a single year. Similar to the study of crystalline silicates, the proposed 7-25 $\mu \mathrm{m}$ survey of the Chamaeleon dark clouds complex will provide many sources, additional to WISE, for follow-up.

\section{Exoplanet Science}

\subsection{Planets Free Floating in Nearby Star Clusters}

The detection of extra-solar planets orbiting other stars over the last decade has revolutionised our understanding of how planets form and the amazing multiplicity of extrasolar planetary systems. We have already found that solar systems that look just like our own are not the norm, and may indeed not be all that common.

One of the primary difficulties in studying planets orbiting other stars is that it is phenomenally difficult to access light that comes from the exoplanets themselves. Typical contrast ratios between gas giant planets and their host star are on the order of $10^{7}$ for very young exoplanets, and up to $10^{10}$ for Solar-age Jupiter-mass planets in Jupiter-like orbits.

This makes it difficult to undertake detailed studies of the chemical and atmospheric properties of exoplanets. Unfortunately, this is a critical gap in our understanding, because at the low temperatures present in exoplanet atmospheres (from $\sim 200$ to $1000 \mathrm{~K}$ ) the complexity of the molecular species that can exist makes constructing robust a priori models for exoplanet spectra difficult. That is, while models can be constructed once we know what an exoplanet's spectrum looks like, it is almost impossible to predict what those spectra will look like in advance. 
One way to make headway in understanding what the spectra of exoplanets will look like is to seek locations where planets can be observed in the absence of a host star, by searching for, and studying, objects of planetary mass 'free floating' in nearby stellar clusters. We know from studies of several nearby star clusters that they host objects as low in mass as a few Jupiter masses. Such objects are bright enough $\left(m_{\mathrm{AB}} \approx 24\right.$ at $K$ band) for detailed study on $8 \mathrm{~m}$ and larger telescopes. PILOT therefore, has an exciting opportunity to use its wide-field near-infrared survey capabilities to probe nearby star clusters to identify their coolest ' $\mathrm{T}$ dwarf' members at temperatures of $500-1000 \mathrm{~K}$.

A few investigators have searched for $\mathrm{T}$ dwarfs in younger (1-10 Myr) stellar environments using traditional optical wide-field techniques (Zapatero Osorio et al. 2002) and narrow-field infrared techniques (Lucas \& Roche 2000; Najita et al. 2000). These have had minimal success with only Zapatero Osorio et al. (2002) claiming the detection of a single $\mathrm{T}$ dwarf. PILOT offers the opportunity to revolutionise this field - first because its unprecedented image quality over wide fields is ideally suited to searching for faint targets in the highly confused low galactic fields where star clusters lie. And secondly, because it will be able to survey the large areas of sky that nearby star clusters subtend (i.e. several $\mathrm{deg}^{2}$ ) down to the magnitudes at which $\mathrm{T}$ dwarfs can be found ( $m_{\mathrm{AB}} \leq 24$ at $K_{\mathrm{d}}$ band). PILOT will be able to map an entire square degree in $J, H$, and $K_{\mathrm{d}}$ to the required depths in around 100 hours. Alternatively, with the provision of suitable methane filters that can detect the unique spectral signature of methane absorption in the $H$ or $K_{\mathrm{d}}$ bands for T dwarfs, a similar time would enable deep direct methane imaging to the same depths, detecting objects as low in mass as a few Jupiter masses, and enabling detailed follow-up studies of these 'planets without stars'.

The southern location of PILOT is an advantage for such work, as the southern sky hosts most of the prime star cluster targets for free-floating planet work — including targets such as R Corona Australis, Lupus, Chameleon, $\rho$ Oph, IC2391, and IC2602.

\subsection{Gravitational Microlensing}

The conditions at Dome $\mathrm{C}$ provide opportunities for studies in planetary microlensing that are not possible at any other site on the globe. By working in conjunction with the existing OGLE-III and MOA-II telescopes in Chile and New Zealand that are dedicated to gravitational microlensing, and also with the MicroFUN and PLANET networks of follow-up telescopes, the PILOT telescope at Dome C would provide the opportunity to make new discoveries of ice-giant and terrestrial planets in the Galaxy. Approximately 150 hours of telescope time on PILOT would be required per annum for 3 to 4 years.

Gravitational microlensing occurs if light rays from a distant star pass sufficiently close to a nearer star so that their path is bent, or lensed, into pairs of images. These images cannot be directly resolved but, as the lensing star moves across the line of sight, the total magnified light from the background star can be measured and this generates a symmetrical light curve profile that is now well recognised (Liebes 1964; Paczynski 1986). If the lens star has a planetary companion, additional lensing may occur, producing a perturbation in the light curve (Liebes 1964; Mao \& Paczynski 1991). This has now been demonstrated on a number of occasions, some of which are referred to below. The first clear demonstration was carried out by the OGLE and MOA groups, where an observed 7 day perturbation in the light curve of a microlensing event was attributed to a 2.6 Jupiter mass planet in an orbit around a 0.63 solar mass main sequence star with an orbital radius of 4.3 AU (Bond et al. 2004; Bennett et al. 2006).

A particularly useful procedure for detecting planets in microlensing events was proposed by Griest \& Safizadeh (1998). They pointed out that if the lens star passes almost directly in front of the background star, so that the magnification caused by lensing is high, then planetary perturbations are very likely to occur at the peak of the event, and these are likely to be of sufficient magnitude to be detectable. In these events of high magnification, the pairs of images caused by lensing combine to form an almost complete Einstein ring, whose radius is typically about 2-3 AU. Gaudi et al. (1998) pointed out that multiple planetary perturbations could be detected in these events, leading to the identification of relatively complex planetary systems. Furthermore, Rattenbury et al. (2002) noted that the critical period for planet detection in these events is the FWHM of the light curve, which typically persists for about 10 hours only. Thus these events need only be intensively monitored over a brief period, during which time they are relatively bright and easily observed. Finally, simulations reveal that planetary perturbations in these events are surprisingly large. Ice-giant planets at orbital radii lying within $\sim 10 \%$ of the Einstein radius yield perturbations of order $0.3 \mathrm{mag}$, easily detectable (Yock 2008). Lighter planets, or planets further from the Einstein ring, yield smaller perturbations, but nevertheless they may be detectable. Heavier planets or planets closer to the ring, yield larger perturbations.

All of the above predictions have now been born out by observation. A planet of mass $3.4 M_{J}$ was observed orbiting a star of mass $\sim 0.46 M_{\odot}$ at $\sim 3.5 \mathrm{AU}$, the most massive planet observed orbiting an M dwarf star (Udalski et al. 2005; Dong et al. 2009). A planet of mass $\sim 13 M_{\oplus}$ was detected orbiting a half solar mass star at $\sim 2.7 \mathrm{AU}$ (Gould et al. 2006). This result, when combined with earlier results, led to the conclusion that approximately one third of all stars have Neptune-like planets. A planetary system was detected with planets of masses $\sim 0.71$ and $\sim 0.27 M_{J}$ at orbital radii $\sim 2.3$ and $\sim 4.6$ AU orbiting a half solar mass star (Gaudi et al. 2008). Most recently, a planet of mass $\sim 3.3 M_{\oplus}$ was detected orbiting a $\sim 0.06 M_{\odot}$ star that is likely to be a brown dwarf (Bennett et al. 2008). Other types of observations have been made in microlensing events of high magnification. Stars have been observed which do not have ice-giant or heavier planets at $\sim 2-3 \mathrm{AU}$ 
(Abe et al. 2004). Also, the shapes of some stars have been measured (Rattenbury et al. 2005). Additional examples of events like those described above are presently under analysis.

However, despite the recent successes of microlensing, there have been a number of missed opportunities where microlensers have been frustrated by poor weather and/or interruptions by daylight at crucial times. PILOT would clearly be ideal for follow-up observations of microlensing events; its location allows uninterrupted coverage of selected events. Also the high cloud-free fraction allows a higher number of events to be monitored and greatly reduces the number of missed opportunities. Furthermore, the seeing is superb, enabling more precise measurements to be made, and the possibility exists for all the critical observations in any one event to be made by a single telescope. This would simplify the analysis of the data, and reduce systematic uncertainties. All the above described successes by microlensing have occurred in observations made towards the galactic bulge, and this presents a high air mass at Dome $\mathrm{C}$, with zenith angles ranging from 44 to $80^{\circ}$ (Yock 2006).

A realistic strategy for Dome $\mathrm{C}$ would be to concentrate on those events for which the peak occurs at zenith angles $\leq 60^{\circ}$ at Dome $\mathrm{C}$, and not to attempt to monitor those at larger zenith angles. Nearly a half of all events should satisfy this criterion. This would provide a sample of at least 30-40 fully-monitored events in a period of 3-4 years, as more than 20 events of high magnification are discovered annually by the OGLE and MOA survey telescopes (Yock 2008). The abundance of Neptune-like planets has already been roughly determined from observations made at midsouthern latitudes (Gould et al. 2006). With the enlarged sample that could be accurately monitored from Dome C, one could anticipate a firm determination of the abundance of ice-giant planets being made in 3-4 years together with the first results on the abundance of terrestrial planets. These measurements would complement corresponding measurements being made by the radial velocity and transit techniques which enjoy greatest sensitivity to planets at small orbital radii.

\subsection{Exoplanet Secondary Transits}

Recently a significant milestone in the field of exoplanets has been achieved: the direct detection of infrared thermal emission of massive planets orbiting close to the star (the so called 'hot Jupiters') via the observation of the secondary eclipse in the wavelength range 3-20 $\mu \mathrm{m}$ (e.g. Agol et al. 2005; Charbonneau et al. 2008). Up to now, all positive detections have been made by the Spitzer satellite.

The observation of multiple exoplanet primary transits and secondary eclipses at infrared wavelengths can allow the determination of high accuracy transit times, which could in principle provide sensitivity to secondary lowermass planets in resonant orbits (Agol et al. 2005); high accuracy transit depths, which can be used to constrain the planet molecular composition using atmosphericabsorption models (Tinetti et al. 2007); accurate eclipse depths, which can be used to determine the planet-tostar flux ratio, and hence the planet thermal emission (Charbonneau et al. 2008); and variations of the eclipse depth, which can be used to infer dynamical circulation regimes in the planet atmospheres (Rauscher et al. 2007). To obtain such data requires a high temporal cadence and a very stable infrared background; Dome $\mathrm{C}$ is thus the only location on the ground at which this science can be achieved.

The specific outcomes of the proposed study are to obtain high-precision photometric light curves in the near and mid-infrared for the secondary transits of all the transiting exoplanets detected in the southern hemisphere. The obtained spectral energy distribution of these hot Jupiters will provide information about the upper atmospheric layers of these planets, most notably the abundance of molecular species and the turbulent mixing with lower layers. Such science thus allows the physical properties of exoplanets to be probed.

Spitzer has provided high precision light curves for many transiting exoplanets, but the detection of secondary transits from the ground has not yet been possible. The Spitzer warm mission could provide 3.6- and 4.5- $\mu \mathrm{m}$ broad-band photometric light curves for every transiting exoplanet. However, no longer wavelength or spectroscopic facility is available. JWST, with an exceptional sensitivity and low thermal background throughout the thermal infrared, combined with a spectroscopic capability, will be an ideal tool for this type of science. However, it is likely that alternatives to JWST will need to be identified to undertake survey projects that require a large amount of observing time.

The primary observing mode for this project would be follow-up pointed observations of previously discovered transiting exoplanets in $L, M$, and $N$ bands with the PILOT PNIRC and PMIRIS cameras. The estimated observing limits for a hot Jupiter around a F5V star are $360 \mathrm{pc}$ ( $L$ band), $190 \mathrm{pc}$ ( $M$ band), and $27 \mathrm{pc}$ ( $N$ band). For a K5V star, the observing limits are $140 \mathrm{pc}$ ( $L$ band), $69 \mathrm{pc}$ ( $M$ band), and $10 \mathrm{pc}$ ( $N$ band). One or two secondary transits would be observed per target. Additional intermediateband filters would be valuable to obtain a higher resolution spectral energy distribution. This target-of-opportunity mode would follow the discovery announcements of major transit survey projects and would be performed throughout the lifetime of the PILOT project.

\section{Solar System and Space Science}

\subsection{Planetary Imaging}

PILOT will be able to obtain exquisite images of planets with resolution comparable with those achieved with the Hubble Space Telescope (see Figure 13). While such imaging is possible for all solar system objects the most valuable scientific application will be the observation of the planets Mars and Venus. These objects cannot be imaged with current adaptive optics systems due to the lack of suitable reference sources (Bailey 2004). Venus 

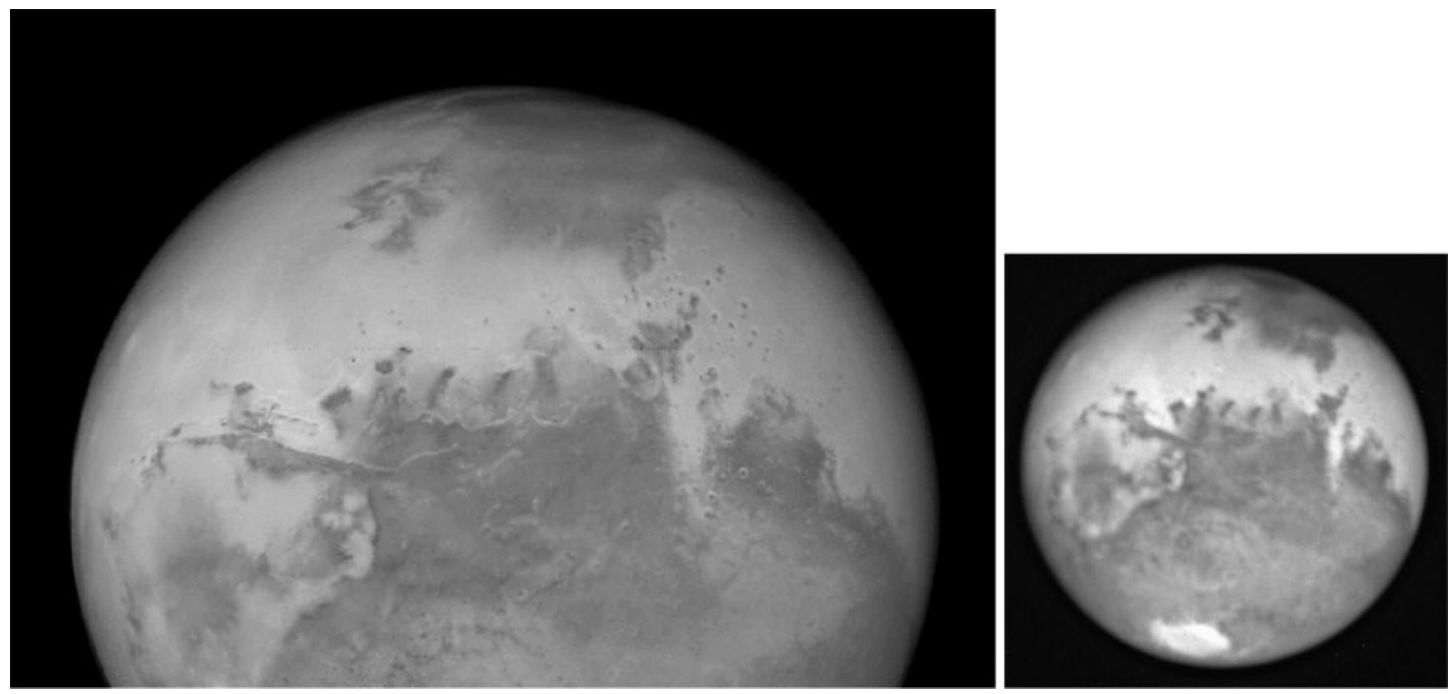

Figure 13 Left: highest resolution image of Mars obtained with HST in Aug 2003. Right: infrared image of Mars obtained at UKIRT in 0.35" seeing using Lucky Imaging. PILOT will improve on the UKIRT image at shorter wavelengths and should obtain images comparable with that from the HST.

cannot be imaged with HST due to pointing constraints, while Mars was difficult to image in the infrared with NICMOS due to saturation.

The Dome $\mathrm{C}$ location means that the best observations will be restricted to times when the planet is at its most southerly declination at opposition (Mars) or conjunction (Venus). However, when these favourable opportunities occur, long durations of visibility are possible. In the best cases, continuous 24-hour observations will be feasible. Venus will have to be observed in daylight, but this should not be a problem owing to its brightness and the reduced daylight sky brightness due to the low aerosol levels.

While these planets are also being studied by orbiting spacecraft, the ability of ground-based observations to provide global continuous coverage provides data that is complementary to the more detailed studies possible from spacecraft. The spatial resolution achieved will be a good match to the resolution of atmospheric general circulation models.

Specific studies, detailed below, that could be carried out on these two planets include the atmospheric super-rotation of Venus, airglow in the upper atmosphere of Venus, circulation and chemistry in the Venus lower atmosphere, and surface pressure on Mars.

The cloud layers of Venus show rotation periods of a few days, a much faster rotation than that of the surface. The origin of this super-rotation is still not fully understood, and is a current topic of much interest for atmospheric circulation modelers (e.g. Lebonnois et al. 2006). Time-resolved imaging observations with high spatial resolution can be used to measure the wind field by tracking the motion of cloud features. Several levels in the Venus atmosphere can be studied, from $50 \mathrm{~km}$ using nightside imaging at 1.7 and $2.3 \mu \mathrm{m}$ to around $70 \mathrm{~km}$ using wavelengths of 365 and $1000 \mathrm{~nm}$ on the dayside. While the ESA Venus Express spacecraft can also do this (e.g. Markiewicz et al. 2007), its imaging observations are limited to the southern hemisphere of Venus by orbital constraints. Current ground-based observations are difficult to use for this purpose because of the limited duration of observations at any one site.

Venus shows intense airglow emission in the singletdelta band of molecular oxygen at $1.27 \mu \mathrm{m}$ that arises at an altitude of $\sim 95 \mathrm{~km}$. The intensity and spatial distribution of this emission varies dramatically from night-to-night (Crisp et al. 1996; Bailey et al. 2008). The airglow could easily be imaged with PILOT using an appropriate narrowband filter, and feature tracking could be used to study the wind field. The region appears to be a chaotic one at the boundary between the retrograde super-rotation seen at the cloud tops, and the sub-solar to anti-solar flow that dominates at high altitudes. Long-term monitoring could be used to investigate whether the pattern swings between these two states as has been suggested from sub-millimetre wind measurements (Clancy, Sandor \& Moriarty-Schieven 2007). Once again PILOT can provide global coverage and continuous visibility not possible with other spacecraft or ground-based systems.

The $\mathrm{CO}$ mixing ratio in the Venus lower atmosphere ( $\sim 30 \mathrm{~km})$ can be measured from nightside observations at around $2.3 \mu \mathrm{m}$. It has been found to show latitudinal variations with a peak at $\sim 60^{\circ}$ latitude and a minimum at the equator (Marcq et al. 2006; Tsang et al. 2007). The distribution is believed to be the result of photochemical formation of $\mathrm{CO}$ above the clouds, a circulation pattern that carries it downwards at $60^{\circ}$ latitude, and chemical removal of $\mathrm{CO}$ as it flows towards the equator. While previous observations have used spectroscopy, modelling has shown that the $\mathrm{CO}$ mixing ratio could be reliably determined from narrow-band filter imaging on and off the $2.3 \mu \mathrm{m}$ CO band. PILOT could therefore be used to obtain long sequences of full-disc images of the $\mathrm{CO}$ distribution that could be used to look for short- or long-term changes in the $\mathrm{CO}$ distribution and hence the circulation patterns. 
Observations of Mars in the $\mathrm{CO}_{2}$ bands can be used to map the distribution of surface atmospheric pressure (Chamberlain, Bailey \& Crisp 2006). While the main effect seen in such observations is the surface topography, long series of observations of this type should also reveal surface pressure effects due to weather systems. Surface pressure is not measured routinely by any of the existing spacecraft. However, it is a key parameter needed to help test and constrain general circulation models of the Martian atmosphere (e.g. Forget et al. 1999).

The observations described above would be obtained with the PLEC and PNIRC instruments on PILOT. Key requirements include the ability to obtain either short exposures and use Lucky Imaging techniques, or a tiptilt correction system capable of operating on the image of a bright planet. For Venus, the telescope must be able to point close to the Sun (at least down to $30^{\circ}$ from the Sun). Additionally, the cameras must not saturate on bright objects such as Mars and Venus (many astronomical cameras do). This either requires the provision of sufficiently short exposure times, or availability of neutral density filters.

\subsection{Solar Coronal Mass Ejections and Solar Flares}

Synchrotron radiations are emitted during solar flares and Sun coronal mass ejections and are usually observed in the microwave part of the spectrum. Understanding this phenomenon will shed light on the physical mechanisms that are responsible for solar flares and coronal mass ejections, such as particle acceleration from the photosphere.

Recent results obtained at sub-millimetre wavelengths have revealed another potential synchrotron radiation spectrum, as illustrated in Figure 14 (Kaufmann et al. 2004). This new spectral component discovered with fluxes increasing for shorter sub-millimetre wavelengths may indicate that the sub-millimetre emission is created by particles accelerated to very high energies.

Current emission models assume three different mechanisms which may become comparable in importance: (a) synchrotron radiation by beams of ultra-relativistic electrons, beams different from the ones producing hard $\mathrm{X}$-rays emissions, (b) synchrotron radiation by positrons produced by nuclear reactions arising from energetic beams interacting at dense regions close to the photosphere, and (c) free-free emission from the chromosphere excited by high-energy electron beams producing hard X-rays emissions (Kaufmann et al. 2006).

Overall, these new results indicate that key questions regarding the physical mechanisms at the origin of solar flares are expected to become better understood with measurements in the far- to mid-infrared range. Submillimetre observations (at 200 and/or $350 \mu \mathrm{m}$ ) of the Sun are proposed here using the SmilePILOT instrument. It could be used either in a monitoring mode or as a follow-up imager if a flare is detected in the visible or ultraviolet.

Current ground-based facilities capable of observing the Sun at sub-millimetre wavelengths, such as the Solar Sub-millimetre Telescope (SST; Kaufmann et al.

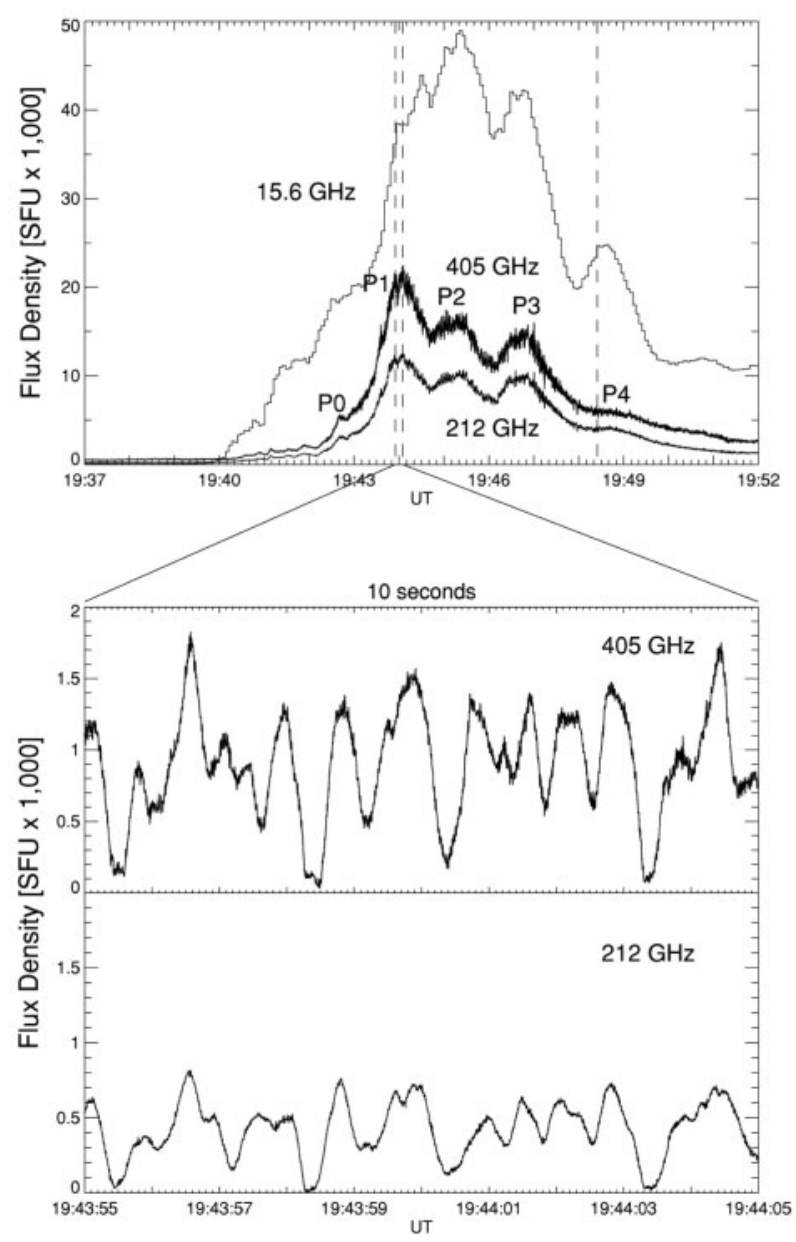

Figure 14 Time profiles of the Solar burst in November 2003. The $405 \mathrm{GHz}$ emission is more intense than the $212 \mathrm{GHz}$ emission, suggesting a possible maximum in the sub-millimetre/far-infrared domain. From Kaufmann et al. (2004).

2002) located in Argentina, are limited by atmospheric conditions. The SST is a $1.5 \mathrm{~m}$ diameter Cassegrain telescope that can observe at $212 \mathrm{GHz}(1.5 \mathrm{~mm})$ and $405 \mathrm{GHz}$ $(750 \mu \mathrm{m})$. The FWHM beams are $2^{\prime}$ and $4^{\prime}$ in these two respective channels. Observations with SST have revealed sub-millimetre bursts in the Solar corona that were associated with large solar flares, as shown in Figure 14. Despite the large Solar flux density in the sub-millimetre ( $>10 \mathrm{kJy}$ ), observations of the Sun from sites such as SST (at an altitude of $2550 \mathrm{~m}$ ) can only be done in the best atmospheric conditions when the atmosphere may become opaque at these wavelengths. Observations at $200 \mu \mathrm{m}$ are basically impossible from the SST site. It is important to obtain observations over a wide wavelength range, from $500 \mu \mathrm{m}$ down to the mid-infrared, in order to understand whether incoherent synchrotron radiations are the main mechanisms that generate sub-millimetre flares. A second limitation of SST is that its small size does not allow high angular resolution observations on the Sun $\left(<1^{\prime}\right)$.

Proposed space projects, such as the Small Explorer for Solar Eruptions (SMESE) project, include instrumentation (the DESIR telescope) operating at both 150 and $35 \mu \mathrm{m}$ (Millard et al. 2006). However, this telescope is 


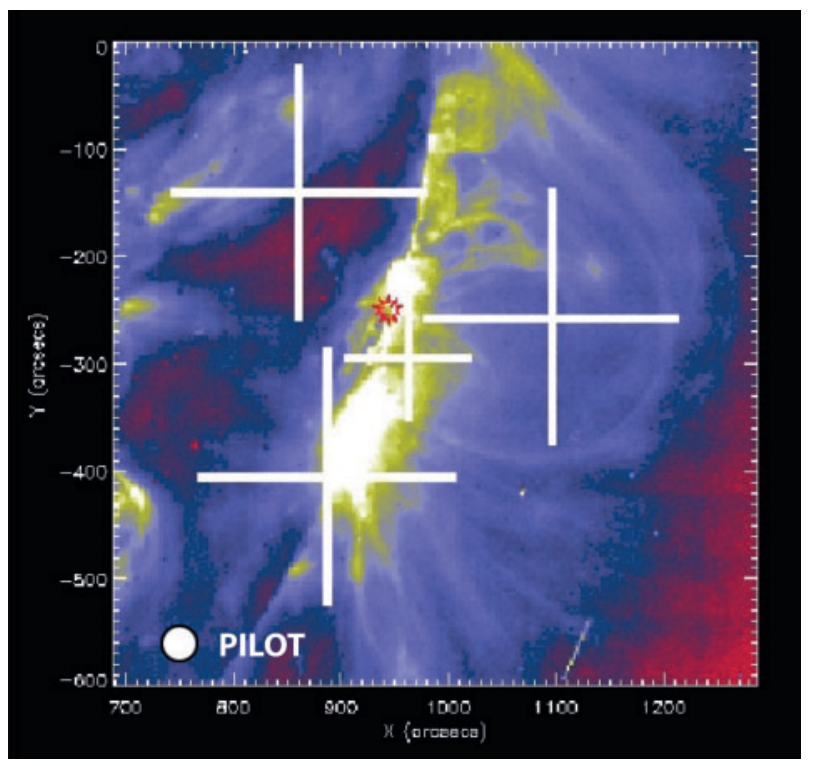

Figure 15 Position and size of the sub-millimetre observing beam of the SST (crosses) on the SOHO ultraviolet image of the Sun taken just before the November 2003 large flare, compared to the size of the PILOT sub-millimetre beam (circle). Adapted from Kaufmann et al. (2004).

relatively small $(\sim 170 \mathrm{~mm})$, and the SMESE bolometers will not be cryogenically cooled. Such a facility is thus limited in terms of time, sensitivity, and resolution. Only major flares with respect to the thermal emission of the Sun will be detected.

Observations of the Sun at Dome C with PILOT can be accomplished at both $30 \mu \mathrm{m}$ and $200 \mu \mathrm{m}$. Given the large flux of the Sun at these wavelengths, observations during the daytime (summer months) with a precipitable water-vapour column density of $0.3 \mathrm{~mm}$ should be possible. Cryogenically cooled detectors should allow detection of minor flares in intensity. Additionally, PILOT would offer a better angular resolution to disentangle the potential sources of flares on the Sun (see Figure 15). A higher angular resolution, and therefore a larger telescope than $\sim 2.5 \mathrm{~m}$ in diameter, is not necessary for observing the Sun.

Complementary observations in the mid-infrared with the PMIRIS instrument on PILOT would also constrain the spectral energy distribution of the incoherent synchrotron radiation. PILOT, as a solar corona mass ejection observer, will be highly complementary to the Murchison Widefield Array (MWA) in Western Australia, which has a key science goal to constrain the magnetic field strength during Sun coronal mass ejections (Salah et al. 2005).

\subsection{Tracking of Space Debris}

There are estimated to be 120000 items $^{9}$ of Low Earth Orbit (LEO) debris larger than $1 \mathrm{~cm}$ and smaller than $10 \mathrm{~cm}$ that are not currently mapped (see Figure 16). This population poses a collision probability that represents a

\footnotetext{
${ }^{9}$ From the ESA Space Debris Mitigation Handbook at www.esa. int/gsp/completed/execsum00_N06.pdf.
}

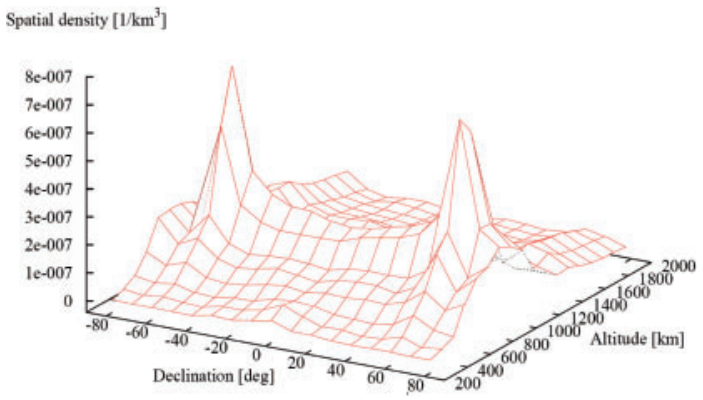

Figure 16 The distribution of the spatial density of LEO debris as a function of altitude and declination, based on the ESA MASTER'99 model for objects larger than $1 \mathrm{~cm}$.

safety risk for manned space-flight activities and a damage risk for spacecraft and satellites. One of the 'advanced technology instrument' concepts for PILOT, the PILOT Satellite Debris Camera (PSDC), contains a $1^{\circ}$ field-ofview with a ring of $1 \mathrm{k} \times 2 \mathrm{k}$ CCD201 L3Vision detectors. This instrument would be capable of identifying accurate orbits for most Low Earth Orbiting space debris larger than $1 \mathrm{~cm}$ within a few years. This would occur at the cost of a small fraction of astronomical observing time, if used in parallel with other instruments.

Sun-synchronous satellites have inclinations of $98.5^{\circ}$, and altitudes of $700-1000 \mathrm{~km}$. They can collide with debris in any orbit at the same altitude. Only debris with inclinations $75-105^{\circ}$ passes over Dome C. For debris with a 100 minute orbit at $850 \mathrm{~km}$ altitude, the angular speed as it passes overhead is 1800 arcsecs per second; the debris thus takes $66 \mathrm{~ms}$ to cross a CCD201 detector. Debris can only be seen when it is in sunlight. At an altitude of $850 \mathrm{~km}$, this means a solar elevation greater than $-28^{\circ}$. Assuming the debris has surface brightness similar to the full moon, a $1-\mathrm{cm}$ satellite at $850 \mathrm{~km}$ subtends 2.4 milli-arcsecs, giving a $V$-band magnitude $m_{\mathrm{AB}}=16.7$. This brightness gives 530 photons on the detector for a crossing time of $66 \mathrm{~ms}$. The debris track has a FWHM width of $\sim 0.2^{\prime \prime}$ and is $120^{\prime \prime}$ long. Assuming a grey sky with brightness $m_{\mathrm{AB}}=19 \mathrm{mag} \operatorname{arcsec}^{-2}$, we get $S N R \approx 7$. Tracks with this $S N R$ would be very obvious on Fourier transforming the images, and the double crossings provide unambiguous confirmation.

Suppose we see a debris trail. Will we be able to catch it again, so as to fully determine the orbit? If the debris is in sun-synchronous orbit, then it will pass roughly overhead 24 hours later. It may be up to 50 minutes (half an orbit) early or late, during which time the rotation of the earth will take Dome $\mathrm{C} \sim 350 \mathrm{~km}$ out of the way, which is a zenith distance of $23.5^{\circ}$ for a height of $850 \mathrm{~km}$. In 24 hours, the position of the satellite is uncertain laterally by $\sim 3 \mathrm{~km}$ or $12^{\prime} \mathrm{rms}$, and in the direction of motion by $3 \mathrm{~km} \mathrm{rms}$, or $\sim 0.5$ seconds in time. So we can predict the crossing 24 hours later well enough to be sure to catch it.

The width of the PILOT field-of-view is $1^{\circ}$, or $14.8 \mathrm{~km}$ at $850 \mathrm{~km}$ altitude. The circumference of the $75^{\circ} \mathrm{S}$ line of latitude is $10000 \mathrm{~km}$. So, given that there are two chances to cross the field-of-view on each orbit, there is a 
$0.3 \%$ chance to see an object (with inclination $>75^{\circ}$ ) on each orbit. There are $\sim 20000$ such objects of size $>1 \mathrm{~cm}$ within the appropriate declination and altitude ranges. The expected discovery rate is then 36 per hour. This number could all be followed up 24 hours later. So if PILOT were used to search for debris for an average of 100 minutes per day for a year, it would (assuming Poisson statistics) see $\sim 66 \%$ of all such objects. A systematic phase-space search pattern could probably be devised to do a much more efficient search. Similar detection rates should be obtained for lower inclination orbits $\left(>65^{\circ}\right)$ observed at higher zenith distance, as although the sky background is higher (leading to a requirement for dark sky conditions) and the image quality worse, the debris takes a longer time to cross the detectors. Thus, in principle, PILOT can obtain orbits for most LEO debris, including almost all objects with inclinations $>65^{\circ}$, in a few years.

\section{Conclusion}

PILOT will be a powerful facility that will allow the detailed investigation of stellar populations in nearby galaxies and stellar clusters. With a suite of instruments comprising a high spatial resolution optical Lucky Imaging camera, a wide field-of-view optical camera with moderate spatial resolution, and a high sensitivity nearinfrared camera, investigations of nearby galaxies at various distances are possible. Three projects are proposed that will examine the Magellanic Cloud galaxies, a sample of Milky Way satellite galaxies, and a sample of Local Group disc galaxies. Additionally, with a stable point spread function and wide-field high-cadence capability, long time-series asteroseismic observations of stellar clusters will be possible for the first time.

The mid-infrared capabilities of PILOT are uniquely suited to the study of star and planet formation within the Galaxy. Firstly, a mid-infrared molecular hydrogen survey of the Central Molecular Zone is proposed; this project, which aims to image a wide region of sky with a high spatial resolution, is not possible with any other telescope. Additionally, studies of circumstellar discs forming around early stage, and embedded, young stellar objects and brown dwarfs, will be possible over wide areas with a high spatial resolution and a broad wavelength coverage; these studies are only possible with the PILOT telescope.

The detection and characterisation of exoplanets can also be addressed with PILOT. With a wide field-of-view and high infrared sensitivity it should be possible to detect free-floating planets in nearby stellar clusters to a low mass limit. The high-cadence capability will allow the characterisation of gravitationally microlensed planets, with a precision not possible with any other single telescope. Finally, a combination of high infrared sensitivity, high cadence, and high atmospheric infrared background stability, will allow the characterisation of transiting hot Jupiters, otherwise only possible with space telescopes such as JWST and Spitzer.

Several projects have been proposed here for PILOT that involve observations within the Solar System. Firstly, the Dome C location, the Lucky Imaging optical camera, and the wide-field near-infrared camera, will enable PILOT to obtain images of planets with a resolution comparable to the Hubble Space Telescope, allowing details of the atmospheric and surface conditions of Mars and Venus to be investigated. Additionally, the potential sub-millimetre capabilities of PILOT will allow it to investigate the physics of solar coronal mass ejections with a higher spatial resolution than other facilities. Finally, if PILOT were equipped with a very wide-field fast imaging camera, its location would allow it to determine the orbits for a large number of small-scale low earth orbit space debris.

\section{Acknowledgments}

The PILOT Science Case, presented here, was produced as part of the PILOT conceptual design study, funded through the Australian Department of Education, Science, and Training through the National Collaborative Research Infrastructure Strategy (NCRIS) scheme, and the University of New South Wales through the UNSW PILOT Science Office. The European contribution has been supported by the ARENA network of the European Commission FP6 under contract RICA26150.

\section{References}

Abe, F. et al., 2004, Sci, 305, 1264

Adams, J. D. et al., 2006, SPIE, 6269, 34

Agabi, A. et al., 2006, PASP, 118, 344

Agol, E., Steffen, J., Sari, R. \& Clarkson, W., 2005, MNRAS, 359,567

Alcalà, J. M. et al., 2008, ApJ, 676, 427

Apai, D. et al., 2005, Sci, 310, 834

Bailey, J., Meadows, V. S., Chamberlain, S. \& Crisp, D., 2008, Icarus, 197,247

Bailey, J., 2004, PASP, 116, 745

Bekki, K. et al., 2007, MNRAS, 377, 215

Bennett, D. P. et al., 2006, ApJ, 647, L171

Bennett, D. P. et al., 2008, ApJ, 684, 663

Benson, A. J., Dzanovic, D., Frenk, C. S. \& Sharples, R. B., 2007, MNRAS, 379, 841

Bond, I. A. et al., 2004, ApJ, 606, L155

Boulanger, F. et al., 2008, ExA, 23, 277

Bower, R. G. et al., 2006, MNRAS, 370, 645

Bullock, J. S. \& Johnston, K. V., 2005, ApJ, 635, 931

Burton, M. G. \& Haas, M. R., 1997, A\&A, 327, 309

Burton, M. G., 1992, AuJPh, 45, 463

Burton, M. G., Hollenbach, D. J. \& Tielens, A. G. G. M., 1992, ApJ, 399,563

Burton, M. G. et al., 1994, PASA, 11, 127

Burton, M. G., Storey, J. W. V. \& Ashley, M. C. B., 2001, PASA, 18,158

Burton, M. G. et al., 2005, PASA, 22, 199

Cambrèsy, L., 1999, A\&A, 345, 965

Chamberlain, S., Bailey, J. \& Crisp, D., 2006, PASA, 23, 119

Charbonneau, D., Knutson, H. A., Barman, T., Allen, L. E., Mayor, M., Megeath, S. T., Queloz, D. \& Udry, S., 2008, ApJ, 686, 1341 Cioni, M. R. et al., 2000, A\&AS, 144, 235

Clancy, R. T., Sandor, B. J. \& Moriarty-Schieven, G. H., 2007, BAAS, 39, 539

Crisp, D., Meadows, V. S., Bézard, B., de Bergh, C., Maillard, J.-P. \& Mills, F. P., 1996, JGR, 101, 4577

Demers, S., Battinelli, P. \& Artigau, E., 2006, A\&A, 456, 905

Dong, S. et al., 2009, ApJ, 695, 970 
Driver, S. P., Allen, P. D., Liske, J. \& Graham, A. W., 2007, ApJ, 657, L85

Eggen, O. J., Lynden-Bell, D. \& Sandage, A. R., 1962, ApJ, 136, 748 Elmegreen, B., 2007, ApJ, 668, 1064

Evans, C. J. et al., 2005, A\&A, 437, 467

Falgarone, E. et al., 2005, EAS, 14, 57

Ferguson, A. M. N., Irwin, M. J., Ibata, R. A., Lewis, G. F. \& Tanvir, N. R., 2002, AJ, 124, 1452

Forget, F. et al., 1999, JGR, 104, 24155

Freyhammer, L. M. et al., 2005, A\&A, 429, 631

Fukugita, M., Hogan, C. J. \& Peebles, P. J. E., 1998, ApJ, 503, 518

Gaudi, B. S. et al., 1998, ApJ, 502, L33

Gaudi, B. S. et al., 2008, Sci, 319, 927

Girardi, L. et al., 2002, A\&A, 391, 195

Gould, A. et al., 2006, ApJ, 644, L37

Griest, K. \& Safizadeh, N., 1998, ApJ, 500, 37

Grundahl, F. et al., 2006, MmSAI, 77, 458

Gullieuszik, M., Held, E., Rizzi, L., Saviane, I., Momany, Y. \& Ortolani, S., 2007, A\&A, 467, 1025

Habart, E. et al., 2005, SSRv, 119, 71

Harris, W. E., 1996, AJ, 112, 1487

Hidas, M. G. et al., 2008, AN, 329, 269

Helmi, A., 2008, A\&ARv, 15, 145

Holtzman, J. A., Smith, G. H. \& Grillmair, C., 2000, AJ, 120,3060

Ibata, R. A., Gilmore, G. \& Irwin, M. J., 1994, Natur, 370, 194

Ibata, R., Martin, N. F., Irwin, M., Chapman, S., Ferguson, A. M. N., Lewis, G. F. \& McConnachie, A. W., 2007, ApJ, 671, 1591

Irwin, M. J., Ferguson, A. M. N., Ibata, R. A., Lewis, G. F. \& Tanvir, N. R., 2005, ApJ, 628, L105

Kato, D. et al., 2007, PASJ, 59, 615

Kauffman, G., Guiderdoni, B. \& White, S. D. M., 1994, MNRAS, 267, 981

Kaufmann, P. et al., 2002, RMxAA, 14, 149

Kaufmann, P. et al., 2004, ApJ, 603, L121

Kaufmann, P. et al., 2006, Solar Active Regions and 3D Magnetic Structure, 26th meeting of the IAU, JD03, 39

Kenyon, S. J. \& Gómez, M., 2001, ApJ, 121, 2673

Kenyon, S. L. \& Storey, J. W. V., 2006, PASP, 118, 489

Kenyon, S. L., Lawrence, J. S., Ashley, M. C. B., Storey, J. W. V., Tokovinin, A. \& Fossat, E., 2006, PASP, 118, 924

Kessler-Silacci, J. et al., 2006, ApJ, 639, 275

Kravtsov, A. V. \& Gnedin, O. Y., 2005, ApJ, 623, 650

Kjeldsen, H. et al., 2009, IAUS, 253, 309

Lawrence, J. S., 2004, PASP, 116, 482

Lawrence, J. S., Ashley, M. C. B., Travouillon, T. \& Tokovinin, A., 2004, Nature, 431, 278

Lawrence, J. S. et al., 2009a, PASA, 26, 379

Lawrence, J. S. et al., 2009b, PASA, 26, 397

Layden, A. C. \& Sarajedini, A., 2000, AJ, 119, 1760

Lebonnois, S., Crespin, A., Hourdin, F., Eymet, V., Fournier, R. \& Dufresne, J.-L., 2006, in European Planetary Science Congress (Berlin), 167

Lee, Y.-W. et al., 1999, Natur, 402, 55

Liebes, S., 1964, PhRvB, 133, 835

Lucas, P. \& Roche, P., 2000, MNRAS, 314, 858

Luhman, K. L., Adame, L., D’Alessio, P., Calvet, N., Hartmann, L., Megeath, S. T. \& Fazio, G. G., 2005, ApJ, 635, L93

Luhman, K. L. et al., 2008a, ApJ, 675, 1375

Luhman, K. L. et al., 2008b, ASPC, Handbook of Star Forming Regions, in press

Maercker, M. \& Burton, M. G., 2005, A\&A, 438, 663

Mainzer, A. K., Eisenhardt, P., Wright, E. L., Liu, F.-C., Irace, W., Heinrichsen, I., Cutri, R. \& Duval, V., 2006, SPIE, 62651

Majewski, S. R. et al., 2005, AJ, 130, 2677

Mao, S. \& Paczynski, B., 1991, ApJ, 374, L37

Marcq, E., Encrenaz, T., Bézard, B. \& Birlan, M., 2006, P\&SS, 54, 1360

Markiewicz, W. et al., 2007, Natur, 450, 633
Millard, A. A. et al., 2006, SPIE, 6266, 15

Mizuno, N. \& Fukui, Y., 2004, ASPC, 317, 59

Mizuno, A. et al., 2001, PASJ, 53, 1071

Molster, F. \& Kemper, C., 2005, SSRv, 119, 3

Morrow, A. L. et al., 2008, ApJ, 676, L143

Mosser, B. \& Aristidi, E., 2007, PASP, 119, 127

Mosser, B. et al., 2007, EAS, 25, 239

Munoz, R. R. et al., 2006, ApJ, 649, 201

Najita, J. et al., 2000, ApJ, 541, 977

Nikolaiev, S. \& Weinberg, M. D., 2000, ApJ, 542, 804

Olsen, K. A. G., Blum, R. D. \& Rigaut, F., 2003, AJ, 126, 452

Paczynski, B., 1986, ApJ, 304, 1

Pancino, E. et al., 2007, ApJ, 661, L155

Parmar, P. S., Lacy, J. H. \& Achtermann, J. M., 1991, ApJ, 372, L25

Parmar, P. S., Lacy, J. H. \& Achtermann, J. M., 1994, ApJ, 430, 786

Persi, P. et al., 2000, A\&A, 357, 219

Persi, P., Marenzi, A. R., Gómez, M. \& Olofsson, G., 2001, A\&A, 376, 907

Persi, P., Marenzi, A. R. \& Gómez, M., 2002, ESA, 511, 221

Persi, P., Marenzi, A. R., Gómez, M. \& Olofsson, G., 2003, A\&A, 399, 995

Pfenniger, D., Combers, F. \& Martinet, L., 1994, A\&A, 285, 79

Pietrzynsky, G. et al., 2008, AJ, 135, 1993

Pigulski, A., 2007, CoAst, 150, 159

Platais, I. et al., 2003, ApJ, 591, L127

Pohlen, M., Erwin, P., Trujillo, I. \& Beckman, J. E., 2008, ASPC, 390, 247

Rattenbury, N. J. et al., 2002, MNRAS, 333, 159

Rattenbury, N. J. et al., 2005, A\&A, 439, 645

Rauer, H., Fruth, T. \& Erikson, A., 2008, PASP, 120, 852

Rauscher, E., Menou, K., Cho, J., Seager, S. \& Hansen, B. M. S. 2007, ApJ, 662, L115

Robertson, B. et al., 2006, ApJ, 645, 986

Robitaille, T. P., Whitney, B. A., Indebetouw, R., Wood, K. \& Denzmore, P., 2006, ApJS, 167, 256

Robitaille, T. P., Whitney, B. A., Indebetouw, R. \& Wood, K., 2007, ApJS, 169, 328

Rodríguez-Fernández, N. J., Martin-Pintado, J., Fuente, A., de Vicente, P., Wilson, T. L. \& Huttemeister, S., 2001, A\&A, 365,174

Salah, J. E., Lonsdale, C. J., Oberoi, D., Cappallo, R. J. \& Kasper, J., 2005, SPIE, 5901, 124

Saunders, W., Gillingham, P., McGrath, A., Haynes, R., Brzeski, J., Storey, J. \& Lawrence, J., 2008a, SPIE, 70124F

Saunders, W., Gillingham, P., McGrath, A., Haynes, R., Storey, J., Lawrence, J., Burton, M. \& Mora, A., 2008b, SPIE, 70144N

Strassmeier, K. G. et al., 2008, A\&A, 490, 287

Tinetti, G. et al., 2007, Natur, 448, 7150

Tomasi, C. et al., 2006, JGR, 111, D20305

Trinquet, H., Agabi, A., Vernin, J., Azouit, M., Aristidi, E. \& Fossat, E., 2008, PASP, 120, 203

Tsang, C. et al., 2007, BAAS, 39

Tully, R. B., 1987, ApJ, 321, 280

Udalski, A., 2005, ApJ, 628, L105

Vazquez-Semadeni, E. et al., 2006, ApJ, 643, 245

Villanova, S. et al., 2007, ApJ, 663, 296

Vlajić, M., Bland-Hawthorn, J. \& Freeman, K. C., 2009, ApJ, 697, 361

Yock, P., 2006, AcASN, 47, 410

Yock, P., 2008, in Proc. Manchester Microlensing Conference, submitted (astro-ph/0805.1775)

Walden, V. P., Town, M. S., Halter, B. \& Storey, J. W. V., 2005, PASP, 117,300

Whitney, B. A. et al., 2008, AJ, 136, 18

Worthey, G., España, A., MacArthur, L. A. \& Courteau, S., 2005, ApJ, 631, 82

Yong, D., Carney, B. W., Teixera de Almeida, M. L. \& Pohl, B. L., 2006, AJ, 131, 2256

Zapatero Osorio, M. R. et al., 2002, ApJ, 578, 536 